\title{
Mechanical fixation by porphyrin connection: Synthesis \& transport studies of a bicyclic dimer
}

Patrick Zwick, ${ }^{a}$ Kevin J. Weiland, ${ }^{a}$ Juraj Malinčík, ${ }^{a}$ Davide Stefani, ${ }^{b}$ Daniel Häussinger, ${ }^{a}$ Herre S. J. v. d. Zant, ${ }^{b}$ Diana Dulićc ${ }^{c}$ and Marcel Mayor ${ }^{\star, a, d, e}$

aDepartment of Chemistry, University of Basel, St. Johanns-Ring 19, 4056 Basel, Switzerland

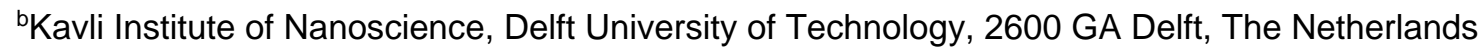
'Department of Physics and Department of Electrical Engineering, Faculty of Physical and Mathematical Sciences, University of Chile, Avenida Blanco Encalada 2008, Santiago 8330015, Chile.

dinstitute for Nanotechnology (INT), Karlsruhe Institute of Technology (KIT), P. O. Box 3640, 76021 Karlsruhe, Germany

eLehn Institute of Functional Materials (LIFM), School of Chemistry, Sun Yat-Sen University (SYSU), 510275 Guangzhou, China

*To whom correspondence should be adressed, marcel.mayor@unibas.ch

\section{Table of Contents}

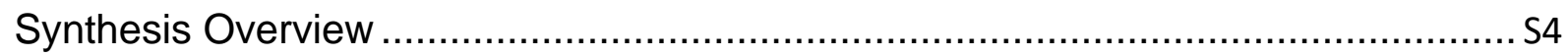

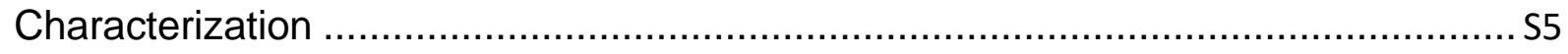

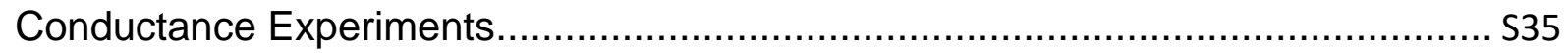

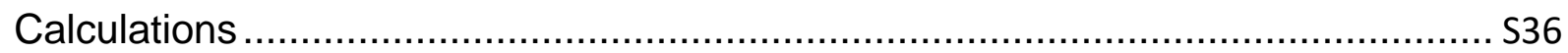

\section{Table of Figures}

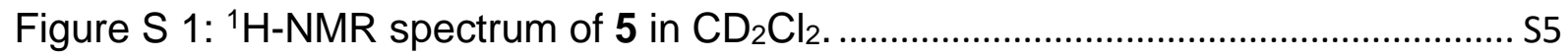

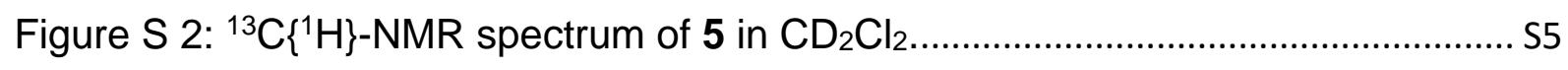

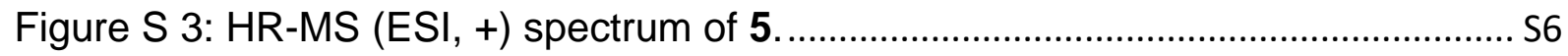




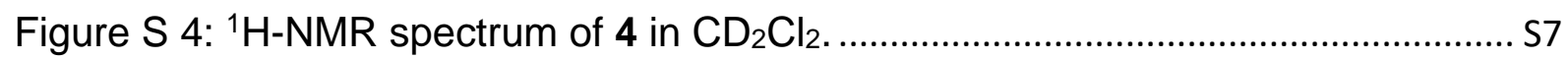

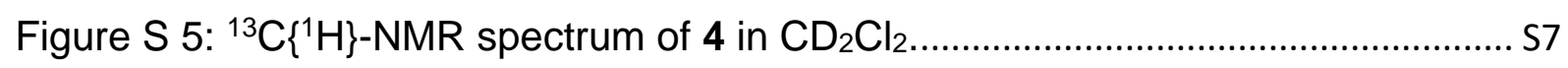

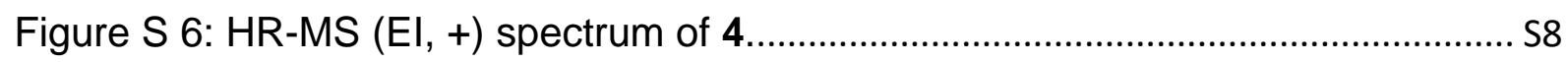

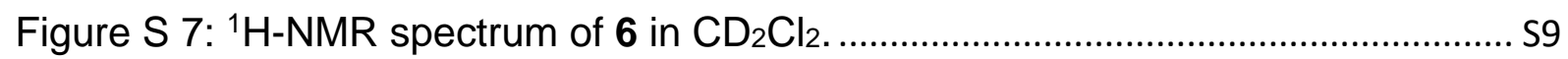

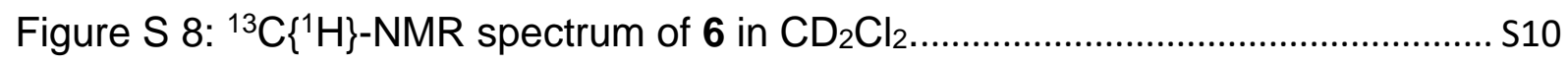

Figure S 9: MALDI-ToF mass spectrum of 6........................................................ S11

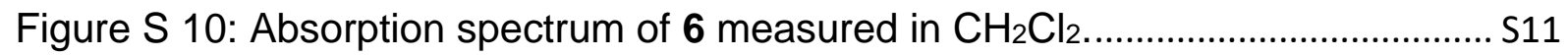

Figure S 11: Order of Elemental Analysis of 6 ...................................................... S12

Figure S 12: Results of Elemental Analysis of 6. ...................................................... S13

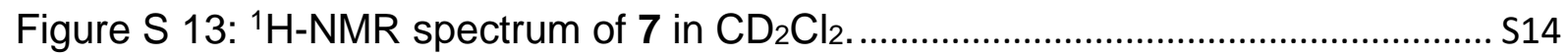

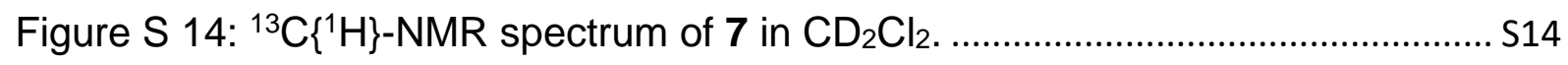

Figure S 15: MALDI-ToF mass spectrum of 7 . .................................................... S15

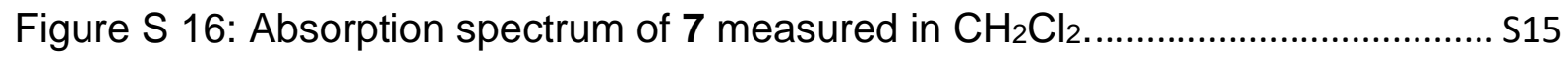

Figure S 17: Order of Elemental Analysis of 7. ....................................................... $\mathrm{S} 16$

Figure S 18: Results of Elemental Analysis of 7. ...................................................... S17

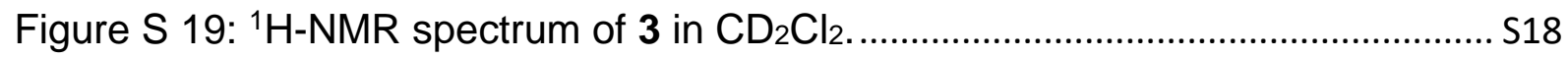

Figure S 20: MALDI-ToF mass spectrum of 3....................................................... S19

Figure S 21: Absorption spectrum of 3 measured in $\mathrm{CH}_{2} \mathrm{Cl}_{2} \ldots \ldots \ldots \ldots \ldots \ldots \ldots \ldots \ldots \ldots \ldots \ldots . . . . . . . . . . . . . . . .19$

Figure S 22: Order of Elemental Analysis of 3. …………………………….......... S20

Figure S 23: Results of Elemental Analysis of 3. .................................................. $\$ 21$

Figure S 24: ${ }^{1} \mathrm{H}-\mathrm{NMR}(600 \mathrm{MHz})$ of 2 dissolved in $\mathrm{C}_{6} \mathrm{D}_{6}$ at $298 \mathrm{~K} \ldots \ldots \ldots \ldots \ldots \ldots \ldots \ldots \ldots . . . . . . . . . . . . . . .22$

Figure S 25: Down-field aromatic region of the ${ }^{1} \mathrm{H}-{ }^{1} \mathrm{H}$ COSY NMR spectrum of 2 in $\mathrm{C}_{6} \mathrm{D}_{6}$ highlighting the singlet $\beta$ proton next to the additional $\mathrm{C}-\mathrm{C}$ bond in red.......... $\mathrm{S} 23$

Figure S 26: Aromatic region of the ${ }^{1} \mathrm{H}-{ }^{13} \mathrm{C}-\mathrm{HSQC}$ spectrum of 2 dissolved in $\mathrm{C}_{6} \mathrm{D}_{6}$ at

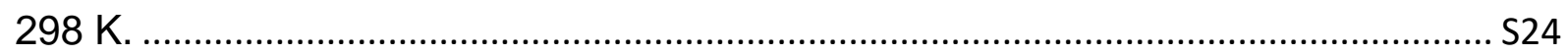

Figure S 27: ${ }^{1} \mathrm{H}-{ }^{-1} \mathrm{H}$-ROESY spectrum of compound $2\left(14.1 \mathrm{~T}, \mathrm{C}_{6} \mathrm{D}_{6}, 298 \mathrm{~K}\right) \ldots \ldots \ldots \ldots . . . \mathrm{S} 25$

Figure S 28: Aromatic region of the ${ }^{1} \mathrm{H}_{-}{ }^{13} \mathrm{C}-\mathrm{HMBC}$ spectrum of compound $\mathbf{2}\left(14.1 \mathrm{~T}, \mathrm{C}_{6} \mathrm{D}_{6}, 298\right.$

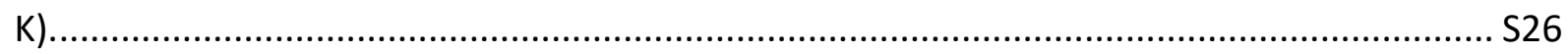

Figure S 29: Two-parameter fit for the PFGSE experiment for the determination of the diffusion coefficient of compound 2 in $\mathrm{C}_{6} \mathrm{D}_{6}$.

Figure S 30: Complete assignment of 2 to proton (black numbers) and carbon (red numbers) chemical shifts. 
Figure S 31: MALDI-ToF mass spectrum of 2. S30

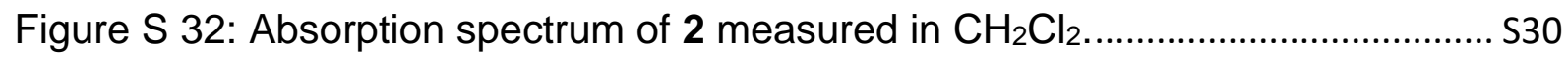

Figure S 33: HR-MS (MALDI-ToF, +) spectrum of 2. ............................................. S31

Figure S 34: MALDI-ToF mass spectrum of 1...................................................... 332

Figure S 35: HR-MS (MALDI-ToF, +) spectrum of 1............................................ S33

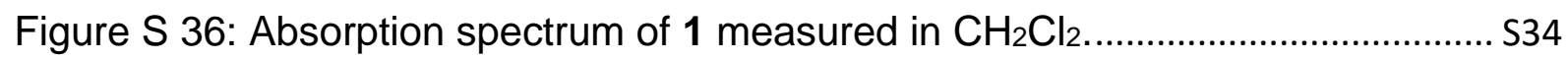

Figure S 37: Single traces that indicate conductance response to mechanical manipulation S35

Figure S 38: Angles and distances of certain atoms in the calculated bowl-shaped structure of 2. S36 


\section{Synthesis Overview}

2<smiles>CC(C)(C)[13C](C)(C)C</smiles>

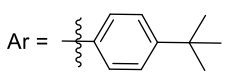

$\underset{81 \%}{\stackrel{\text { ii) }}{\longrightarrow}}$<smiles>C=C(C)C(C(C)C)(C(C)C)C(C)(C)C</smiles>

2

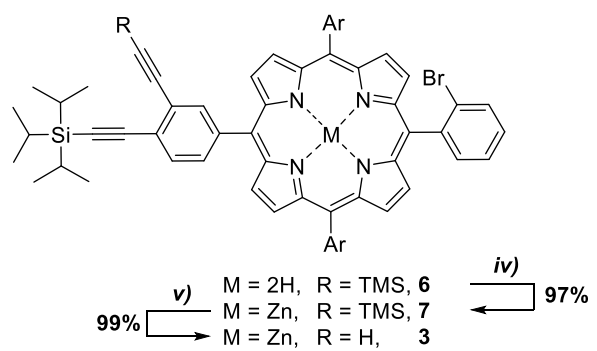

vi)
$5 \%$

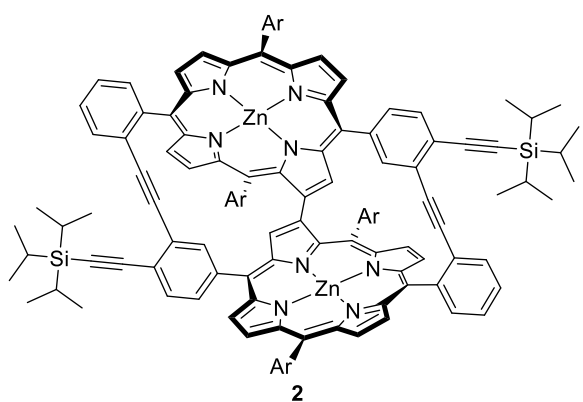

Scheme $S$ 1: Synthesis Overview to 1. : i) Ethynyltrimethylsilane, $\operatorname{Pd}\left(\mathrm{PPh}_{3}\right)_{2} \mathrm{Cl}_{2}$, Cul, piperidine/THF (1:3), rt, $3 \mathrm{~h}$; ii) Ethynyltriisopropylsilane, $\mathrm{Pd}\left(\mathrm{PPh}_{3}\right)_{2} \mathrm{Cl}_{2}$, Cul, piperidine/toluene (1:3), $80{ }^{\circ} \mathrm{C}, 2 \mathrm{~h}$; iii) 1) $\mathrm{BF}_{3} \cdot \mathrm{OEt}_{2}, \mathrm{CH}_{2} \mathrm{Cl}_{2}$, $10{ }^{\circ} \mathrm{C}$, 15 min, 2) $\mathrm{DDQ}, \mathrm{CH}_{2} \mathrm{Cl}_{2}$, rt, 30 min.; iv)) $\mathrm{Zn}(\mathrm{OAc})_{2}, \mathrm{CH}_{2} \mathrm{Cl}_{2} / \mathrm{CH}_{3} \mathrm{OH}$ (1:1), rt, 1 h; v) $\mathrm{K}_{2} \mathrm{CO}_{3}, \mathrm{CH}_{2} \mathrm{Cl}_{2} / \mathrm{CH}_{3} \mathrm{OH}$ (1:1), rt, 2 h; vi) $\mathrm{Pd}\left(\mathrm{PPh}_{3}\right)_{4}, \mathrm{dppf}$, Cul, NEt $/$ /toluene (1:3), $110^{\circ} \mathrm{C}, 2 \mathrm{~d}$; vii) 1) TBAF, THF, rt, $\left.1 \mathrm{~h}, 2\right) \mathrm{S}$-(4iodophenyl) ethanethioate, $\mathrm{Pd}\left(\mathrm{PPh}_{3}\right)_{4}, \mathrm{Cul} \mathrm{NEt}_{3} / \mathrm{THF}(1: 3), \mathrm{rt}, 2 \mathrm{~h}$. 


\section{Characterization}

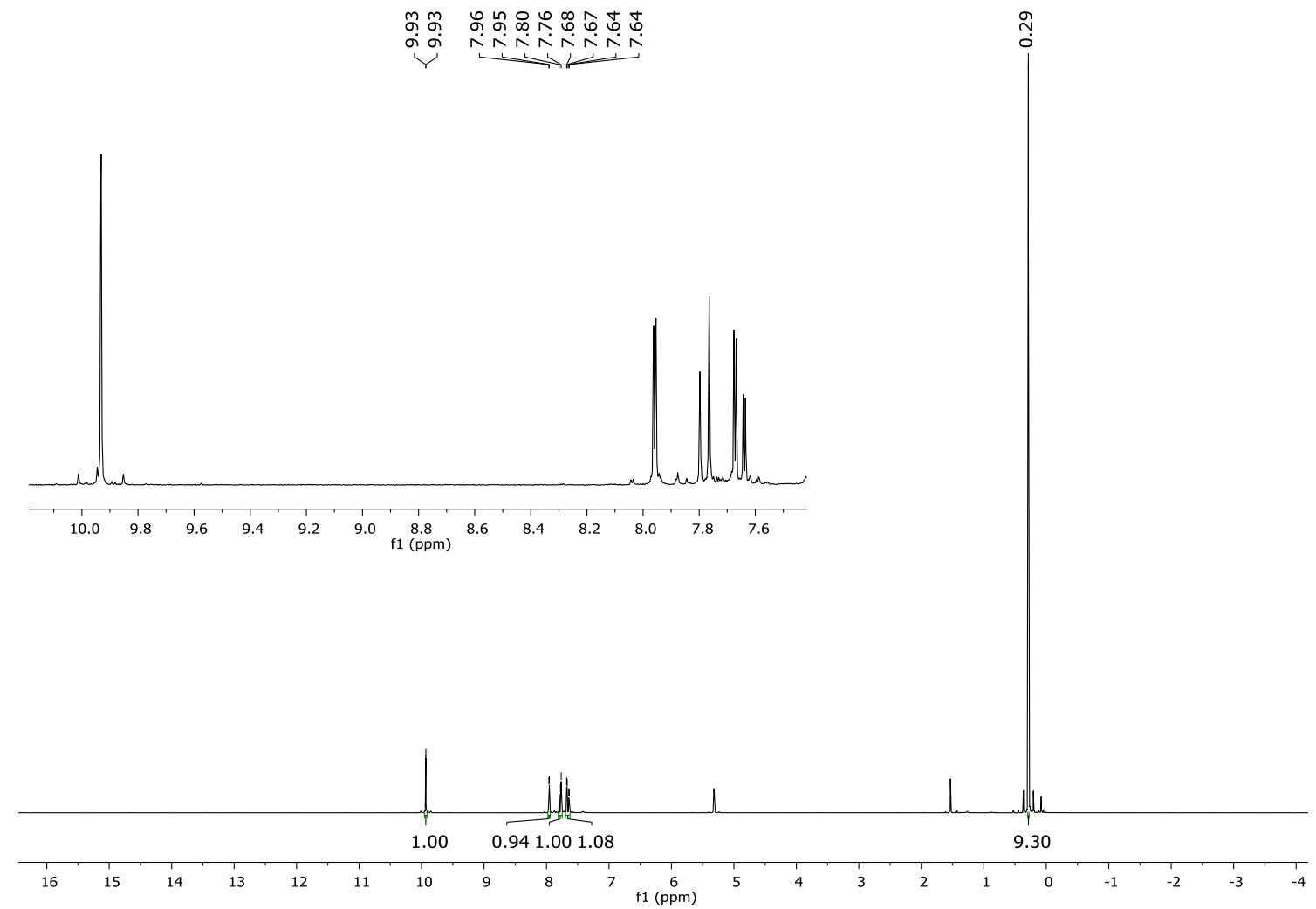

Figure $S$ 1: ${ }^{1} \mathrm{H}-\mathrm{NMR}$ spectrum of 5 in $\mathrm{CD}_{2} \mathrm{Cl}_{2}$.

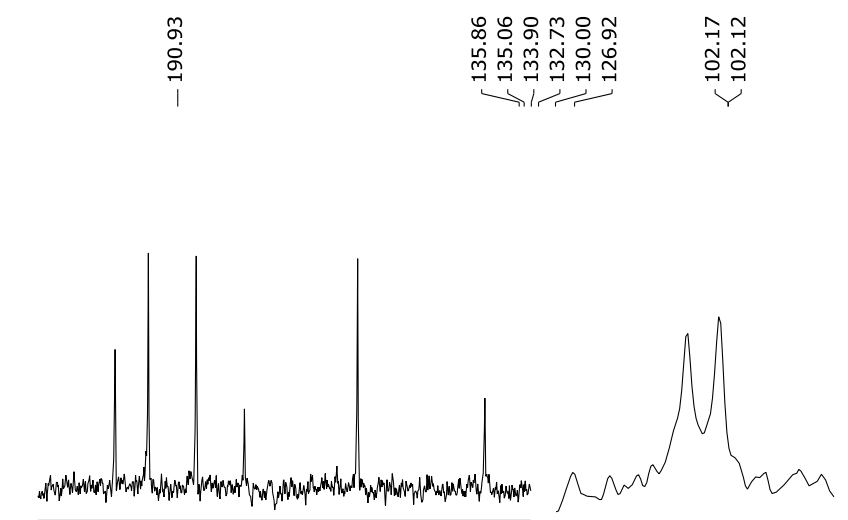

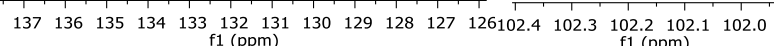

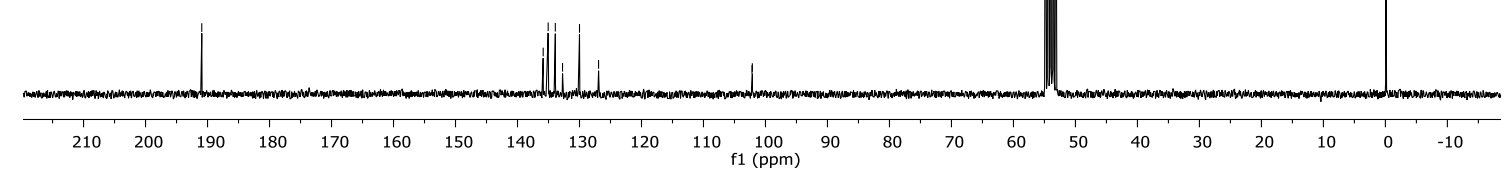

Figure $S$ 2: ${ }^{13} \mathrm{C}\left\{{ }^{1} \mathrm{H}\right\}-\mathrm{NMR}$ spectrum of 5 in $\mathrm{CD}_{2} \mathrm{Cl}_{2}$. 


\title{
Mass Spectrum SmartFormula Report
}

Analysis Info

Analysis Name E:lacq data for data analysislzwp309 001.d

Method

Sample Name

Comment

21 Direct pos low.m

Patrick Zwick

Acquisition Date 21.11.2017 15:38:39

Operator $\quad \mathrm{hn}$

Instrument / Ser\# maXis 4G

21243
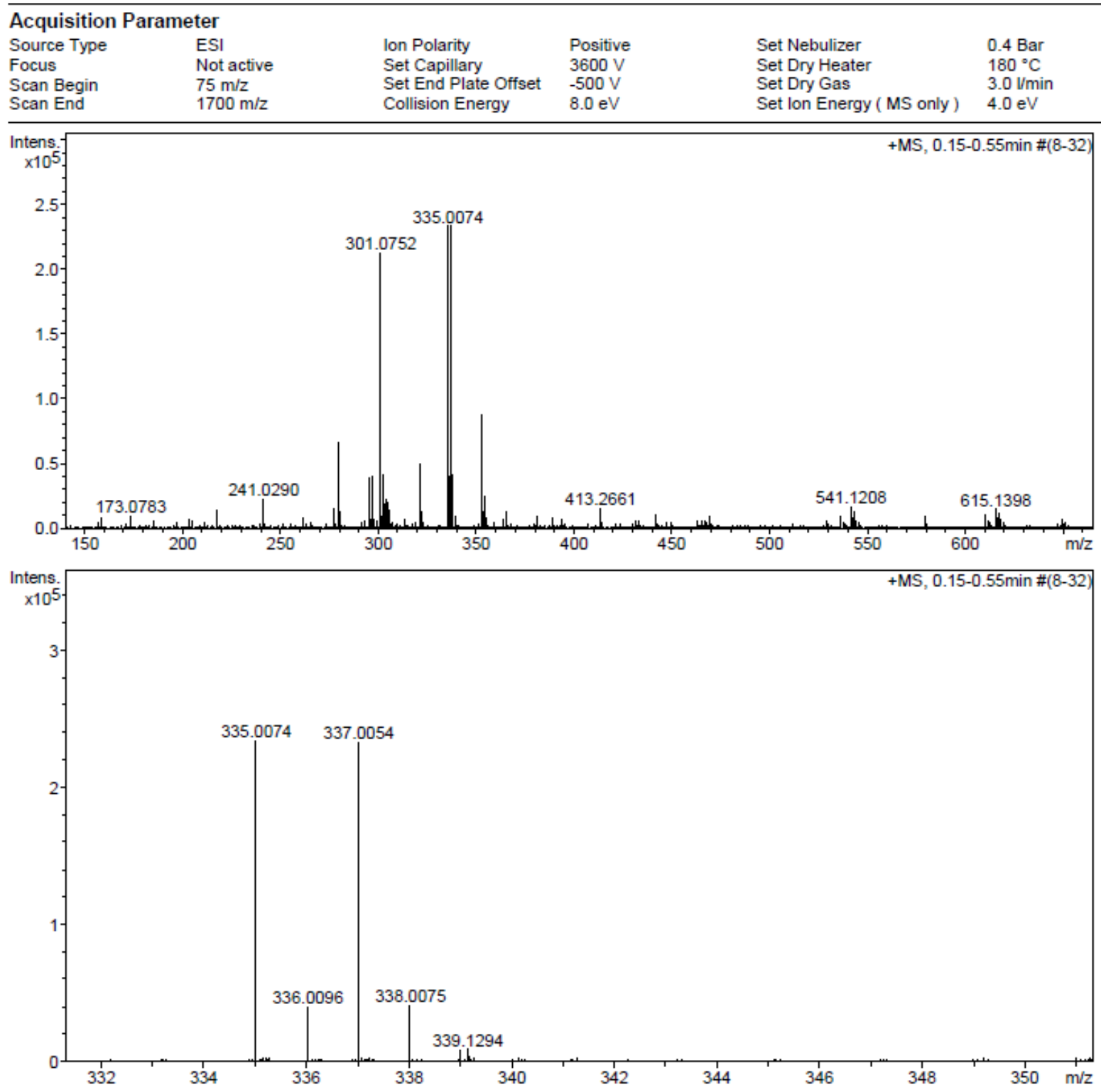

Meas. $\mathrm{m} / \mathrm{z}$ \# Formula

335.0074

$\begin{array}{lrr}\mathrm{C} 13 \mathrm{H} 17 \mathrm{BrNa} O 2 \mathrm{Si} & 100.00 & 335.0073\end{array}$

$\mathrm{m} / \mathrm{z}$

\begin{abstract}
-0.
\end{abstract}
$-0.1$

[ppm]

Sigma rdb $\mathrm{e}^{-}$Conf $z$

Figure S 3: HR-MS (ESI, +) spectrum of 5. 


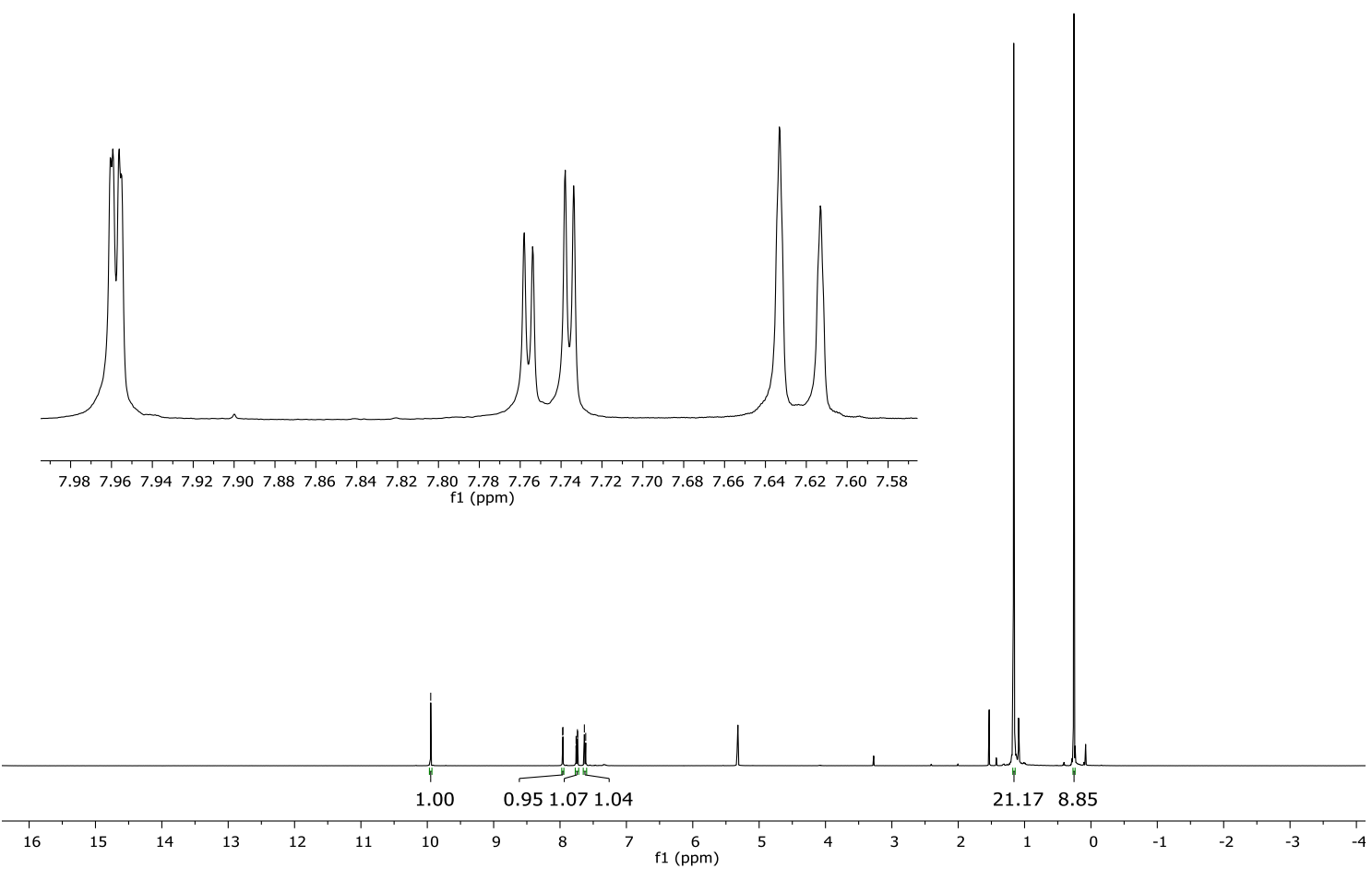

Figure S 4: ${ }^{1} \mathrm{H}-\mathrm{NMR}$ spectrum of 4 in $\mathrm{CD}_{2} \mathrm{Cl}_{2}$.

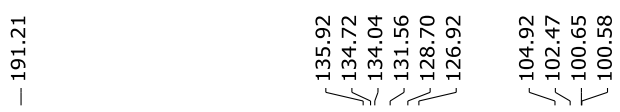

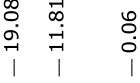

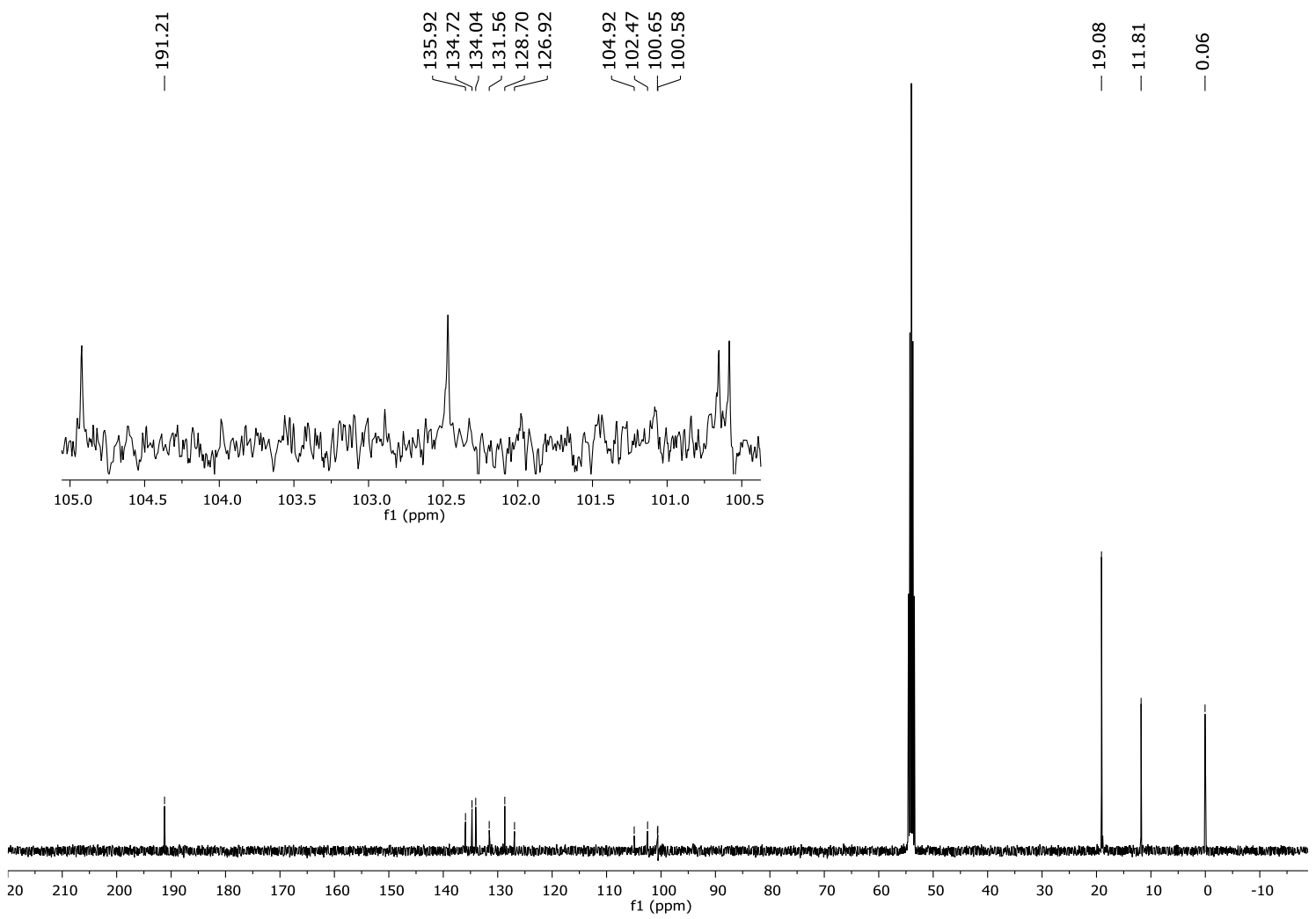

Figure $S 5:{ }^{13} \mathrm{C}\left\{{ }^{1} \mathrm{H}\right\}-\mathrm{NMR}$ spectrum of 4 in $\mathrm{CD}_{2} \mathrm{Cl}_{2}$. 


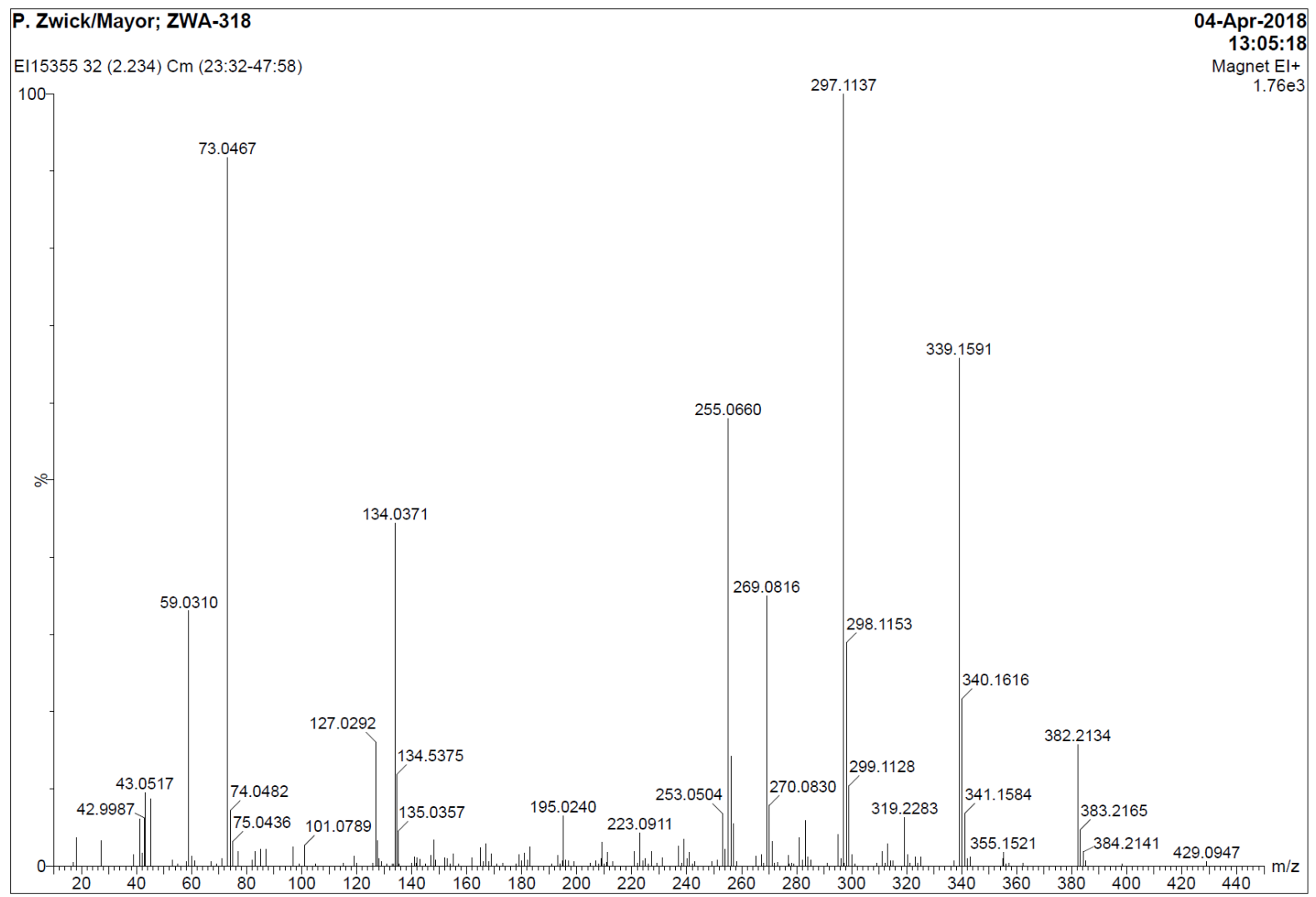

Figure S 6: HR-MS $(\mathrm{El},+)$ spectrum of 4. 


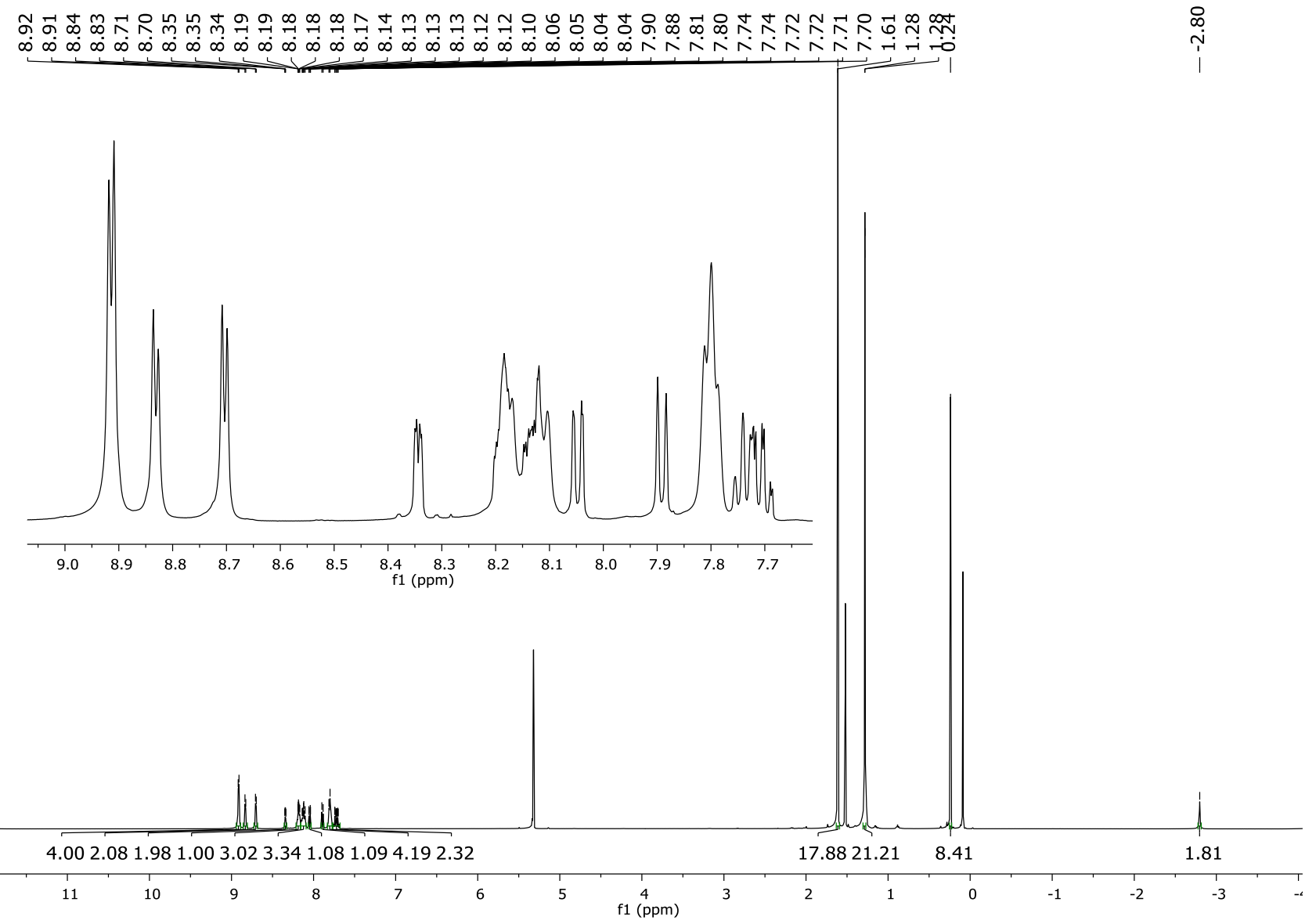

Figure $S$ 7: ${ }^{1} \mathrm{H}-\mathrm{NMR}$ spectrum of 6 in $\mathrm{CD}_{2} \mathrm{Cl}_{2}$. 

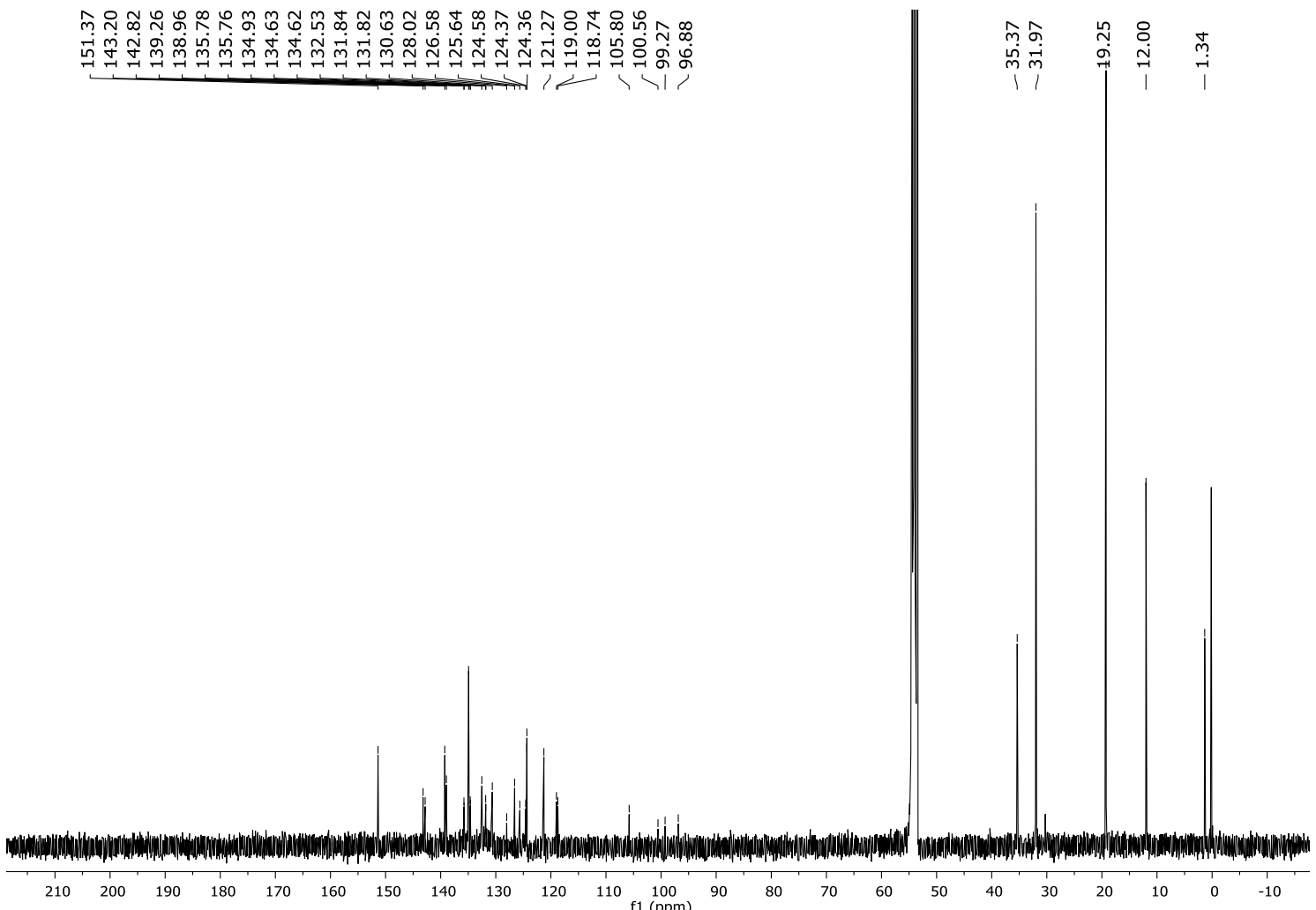

Figure $S 8:{ }^{13} \mathrm{C}\left\{{ }^{1} \mathrm{H}\right\}-\mathrm{NMR}$ spectrum of 6 in $\mathrm{CD}_{2} \mathrm{Cl}_{2}$. 


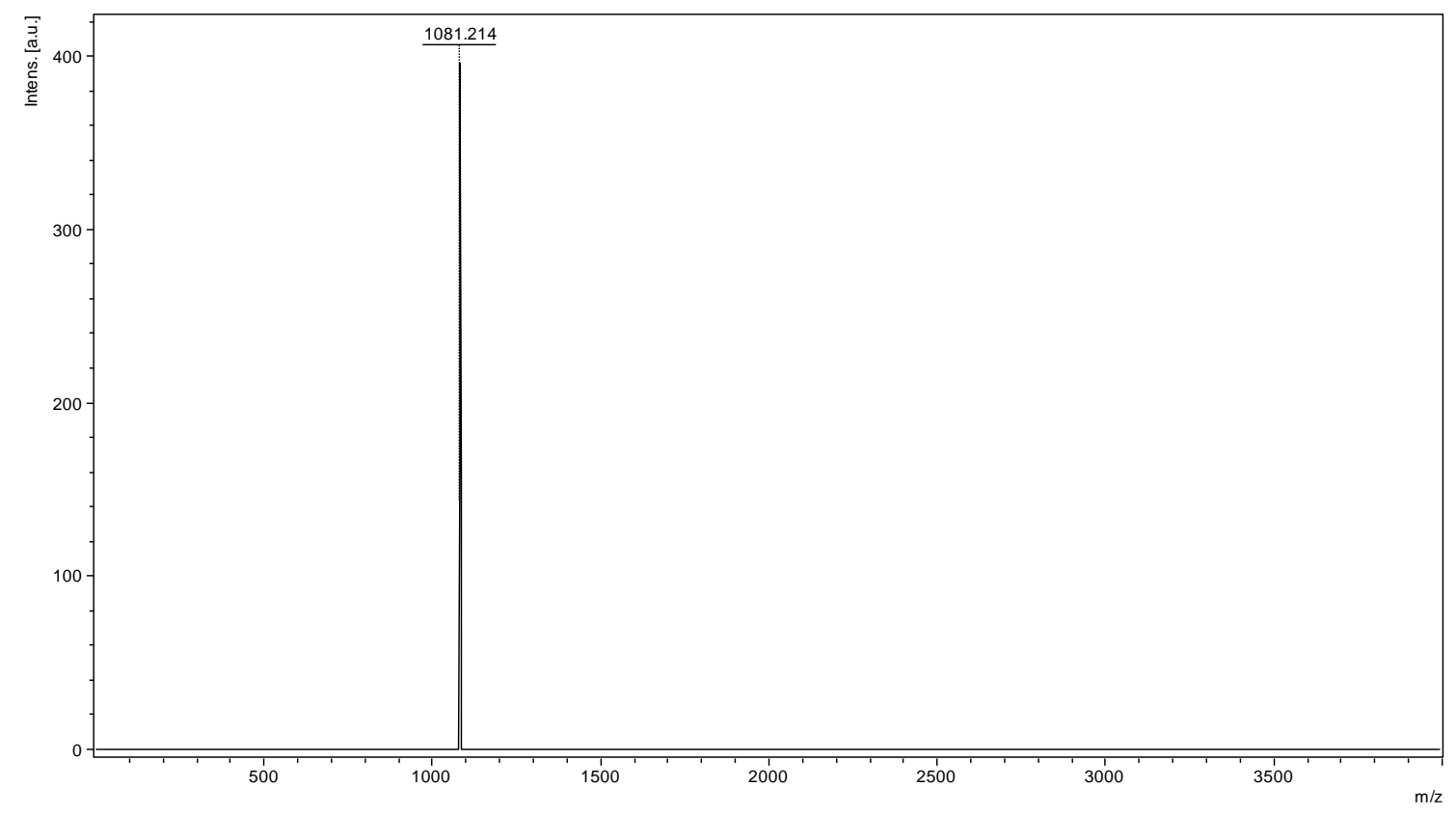

Figure S 9: MALDI-ToF mass spectrum of 6.

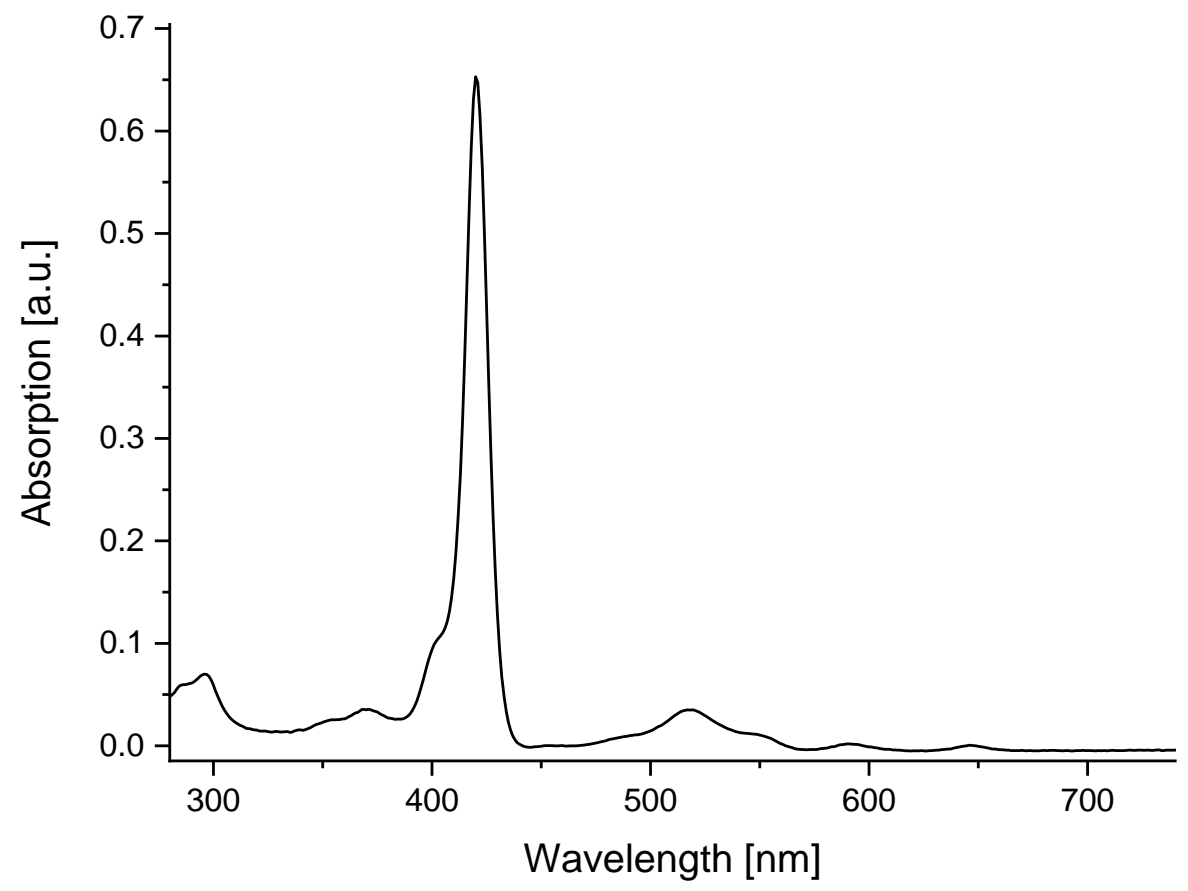

Figure S 10: Absorption spectrum of 6 measured in $\mathrm{CH}_{2} \mathrm{Cl}_{2}$. 


\section{Customer}

\begin{tabular}{|l|l|}
\hline Name: Patrick Zwick & Date:13.11.17 \\
\hline Institute: org. Chemie & Tel: 70787 \\
\hline Labor:OC8 & Email:patrick.zwick@unibas.ch \\
\hline
\end{tabular}

Email supervisor: marcel.mayor@unibas.ch

\section{Sample}

\begin{tabular}{|c|c|}
\hline \multicolumn{2}{|l|}{ Sample name:zwp303 } \\
\hline \multicolumn{2}{|c|}{ Aspect / texture: dark purple microcrystalline solid } \\
\hline Molecular formula: $\mathrm{C} 68 \mathrm{H} 73 \mathrm{BrN} 4 \mathrm{Si} 2$ & Molar mass: 1080.456 (exact mass) \\
\hline
\end{tabular}

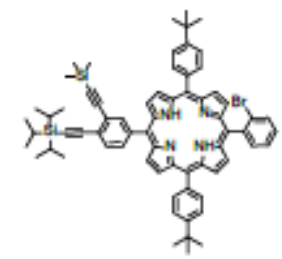

Chemical Formula: $\mathrm{C}_{20} \mathrm{H}_{72} \mathrm{BrN}$

Eract Masa: 1080.455

Eemental Analyals: C, $75.45 ; \mathrm{H}, 6.8 \mathrm{D} ; \mathrm{Br}, 7.38 ; \mathrm{N}, 5.18 ; 8,5.19$

\section{Sample details}

Is the sample harmful? (skin, lungs, toxic)

Is the sample light sensitive?

Is the sample electrostatic?

Is the sample hygroscopic?

Do you want your sample back?

Additional informations:

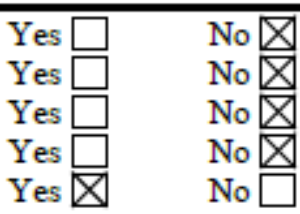

$\begin{array}{ll}\text { Yes } \square & \text { No } \square \\ \text { Yes } \square & \text { No } \square\end{array}$

Figure S 11: Order of Elemental Analysis of 6 


\section{Elemental analysis}

Sample name:zwp303

Single determination $\bigotimes$

Double determination $\square$

\begin{tabular}{|c|c|c|}
\hline C $\quad$ D & $\mathrm{H}$ 区 & $\mathrm{N} \quad \square$ \\
\hline
\end{tabular}

\section{Results}

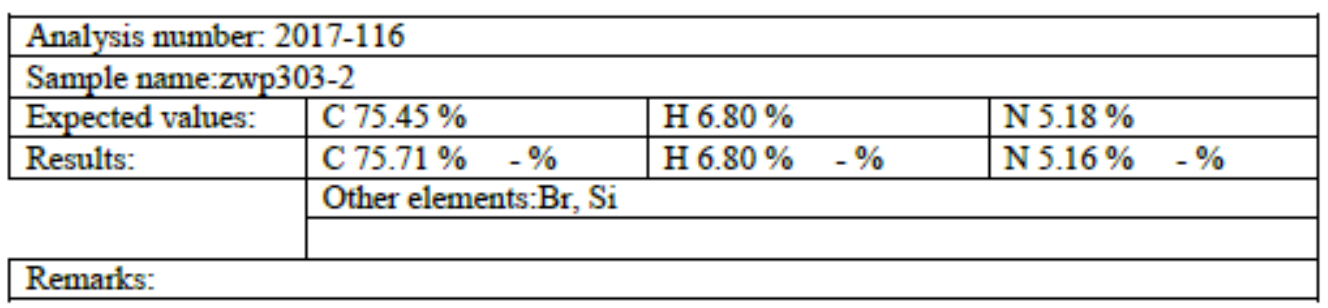

Figure S 12: Results of Elemental Analysis of 6. 


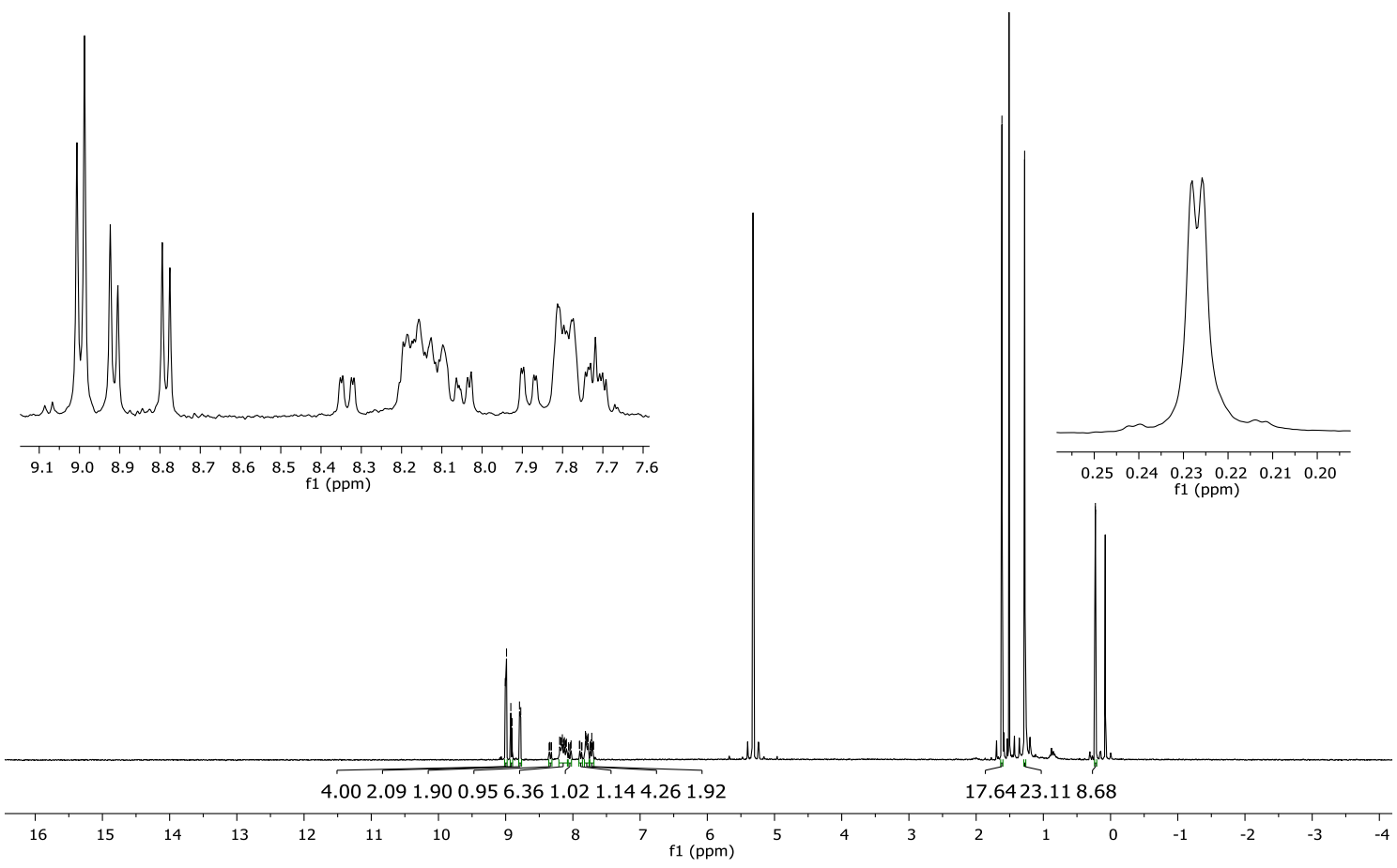

Figure $S$ 13: ${ }^{1} \mathrm{H}-\mathrm{NMR}$ spectrum of 7 in $\mathrm{CD}_{2} \mathrm{Cl}_{2}$.

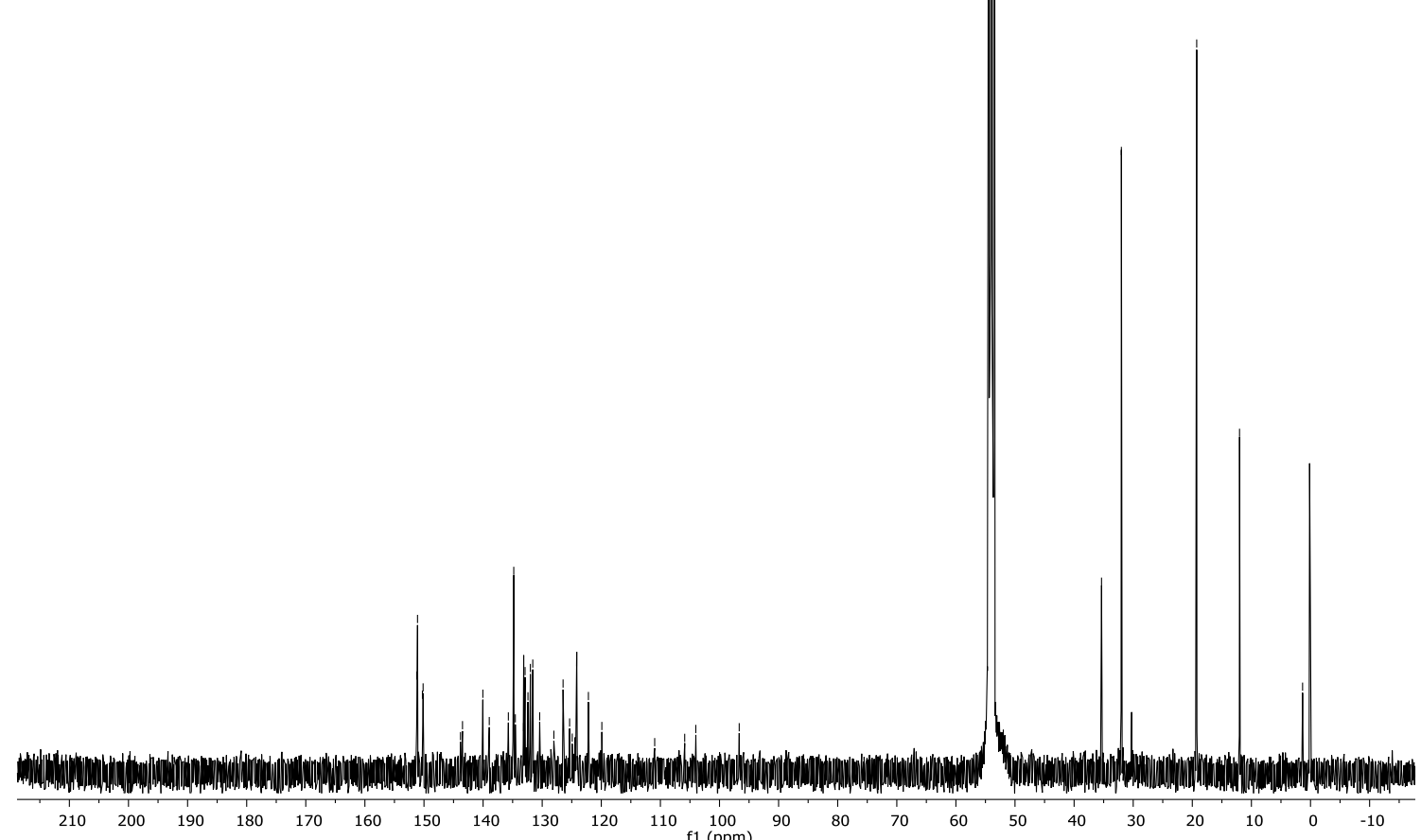

Figure $S$ 14: ${ }^{13} \mathrm{C}\left\{{ }^{1} \mathrm{H}\right\}-\mathrm{NMR}$ spectrum of 7 in $\mathrm{CD}_{2} \mathrm{Cl}_{2}$. 


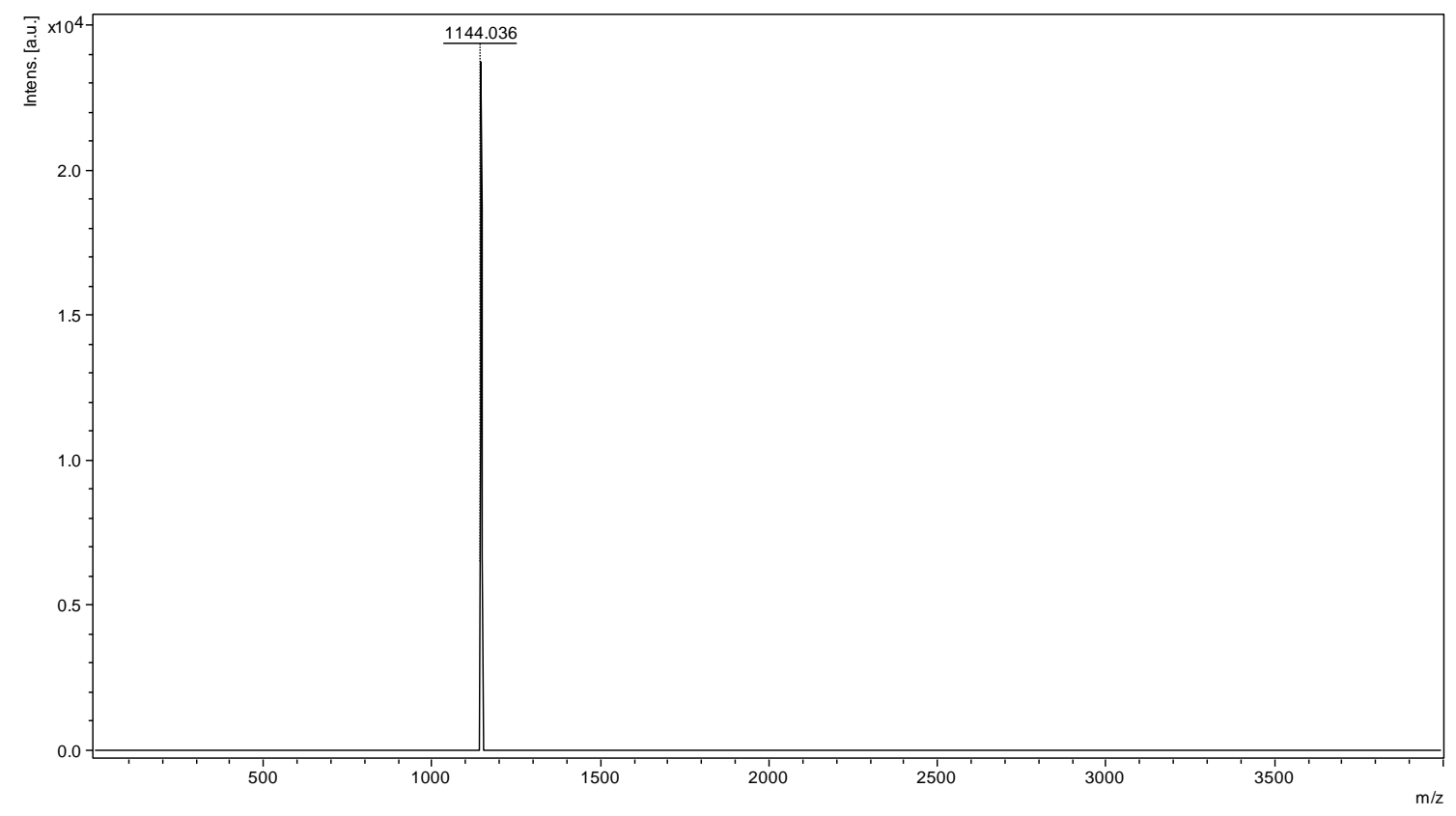

Figure $S$ 15: MALDI-ToF mass spectrum of 7.

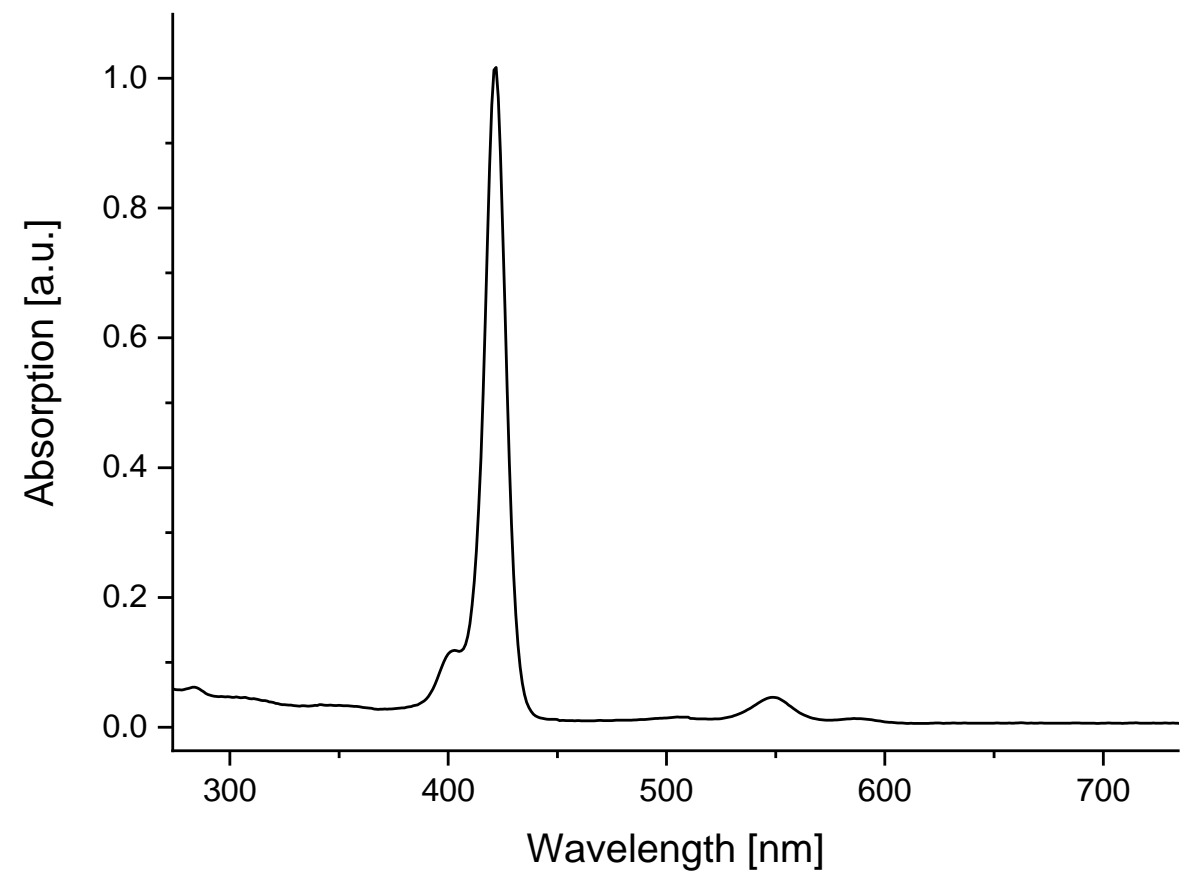

Figure S 16: Absorption spectrum of 7 measured in $\mathrm{CH}_{2} \mathrm{Cl}_{2}$. 


\section{Customer}

\begin{tabular}{|l|l|}
\hline Name: Patrick Zwick & Date:13.11.17 \\
\hline Institute: org. Chemie & Tel: 70787 \\
\hline Labor:OC8 & Email:patrick.zwick@unibas.ch \\
\hline
\end{tabular}

Email supervisor: marcel.mayor@unibas.ch

\section{Sample}

Sample name:zwp305

Aspect / texture: dark purple microcrystalline solid

\begin{tabular}{|l|l|l}
\hline Molecular formula:C68H71BrN4Si2Zn & Molar mass: 1142.369 (exact mass)
\end{tabular}

Used solvent: toluene, $\mathrm{dcm}$, chloroform

Structure:

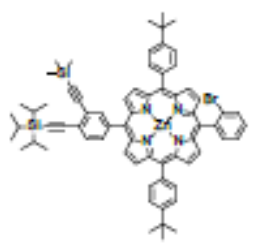

Chemical Formula: $\mathrm{C}_{00} \mathrm{H}_{7}, \mathrm{BrN}_{4}, \mathrm{Bl}_{2} \mathrm{Zn}$

Eract Mess: 1142.369

Eementa Anaysa: $\mathrm{C}, 71.28 ; \mathrm{H}, 6.25 ; \mathrm{Br}, 6.97 ; \mathrm{N}, 4.89 ; 81,4.90 ; \mathrm{Zn}, 5.71$

\section{$\underline{\text { Sample details }}$}

Is the sample harmful? (skin, lungs, toxic)

Is the sample light sensitive?

$\begin{array}{ll}\text { Yes } \square & \text { No } \square \\ \text { Yes } \square & \text { No } \square \\ \text { Yes } \square & \text { No } \square \\ \text { Yes } \square & \text { No } \square \\ \text { Yes } \square & \text { No } \square\end{array}$

Is the sample electrostatic?

Is the sample hygroscopic?

Do you want your sample back?

Additional informations:

Figure S 17: Order of Elemental Analysis of 7. 


\section{Elemental analysis}

Sample name:zwp305

Single determination $\bigotimes$

Double determination

C $\mathrm{Q}$

Other elements: $\mathrm{Br}, \mathrm{Si}, \mathrm{Zn}$

\begin{tabular}{l|l}
$\mathrm{H}$ Q & $\mathrm{N}$ Q
\end{tabular}

\section{$\underline{\text { Results }}$}

\begin{tabular}{|c|c|c|c|}
\hline \multicolumn{4}{|c|}{ Analysis number: $2017-117$} \\
\hline \multicolumn{4}{|c|}{ Sample name:zwp305 } \\
\hline Expected values: & C $71.28 \%$ & H $6.25 \%$ & $\mathrm{~N} 4.89 \%$ \\
\hline \multirow[t]{2}{*}{ Results: } & C $71.43 \% \quad-\%$ & H $6.44 \% \quad-\%$ & N $4.82 \% \quad-\%$ \\
\hline & \multicolumn{3}{|c|}{ Other elements: $\mathrm{Br}, \mathrm{Si}, \mathrm{Zn}$} \\
\hline
\end{tabular}

Figure S 18: Results of Elemental Analysis of 7. 


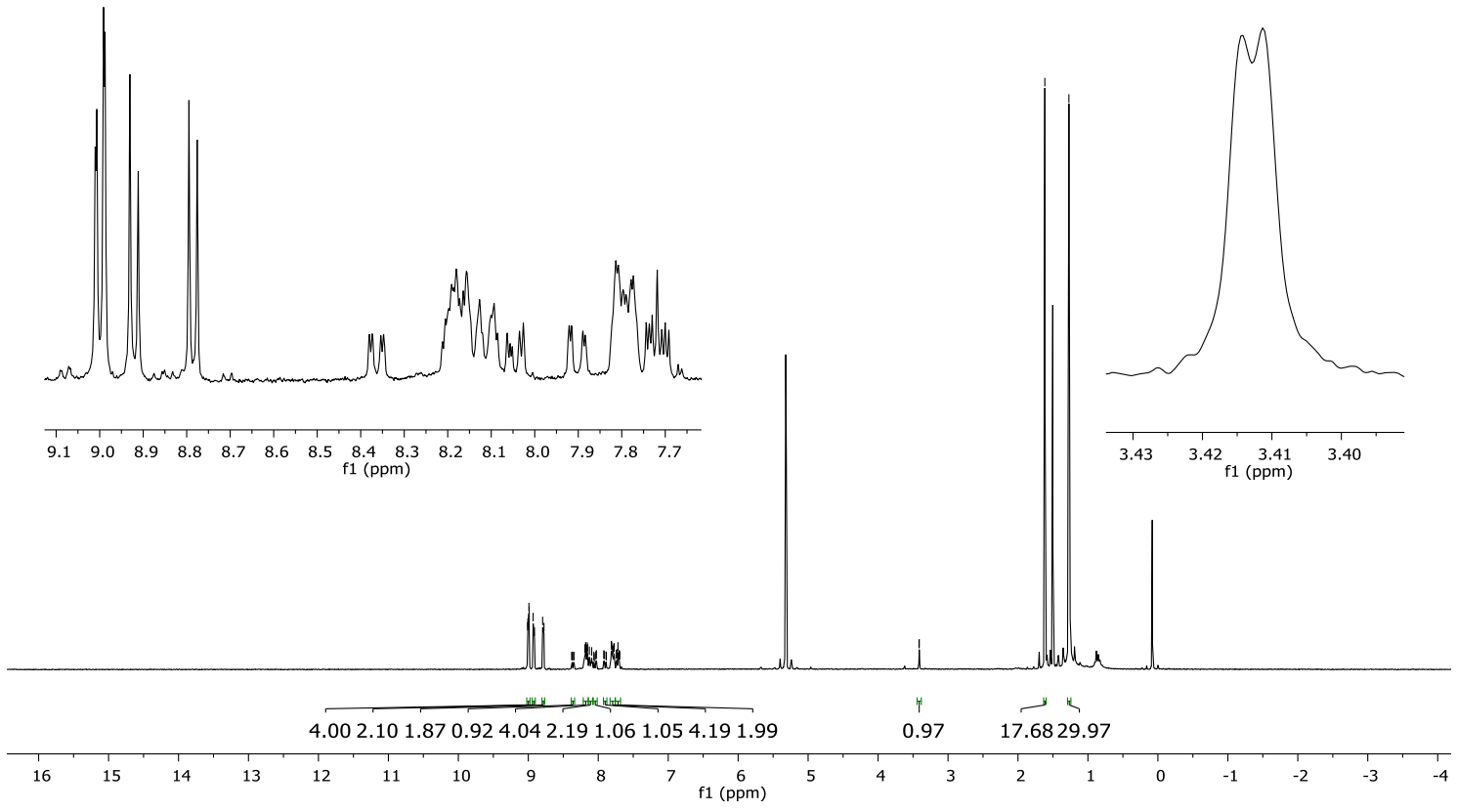

Figure $S$ 19: ${ }^{1} \mathrm{H}-\mathrm{NMR}$ spectrum of 3 in $\mathrm{CD}_{2} \mathrm{Cl}_{2}$. 


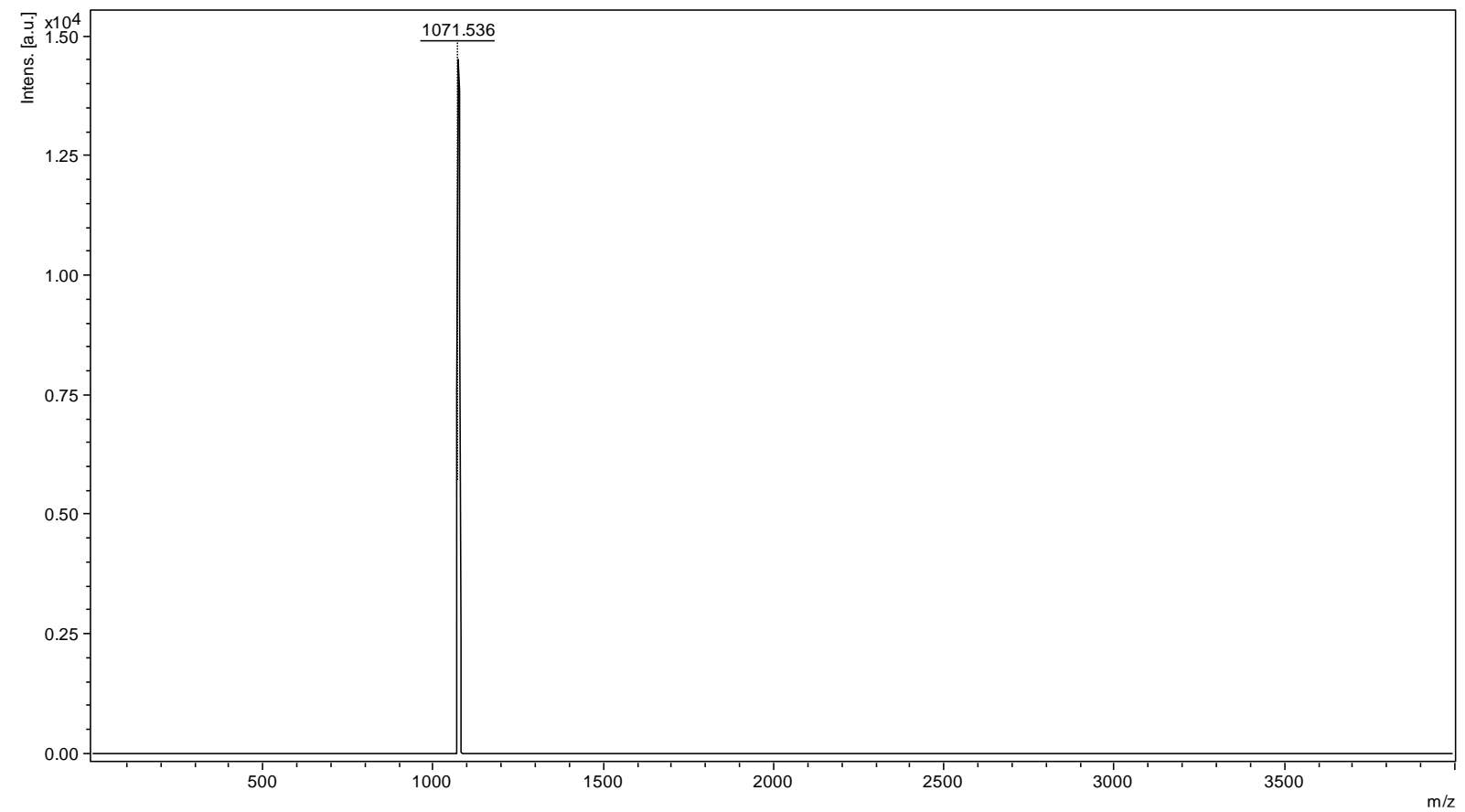

Figure S 20: MALDI-ToF mass spectrum of 3.

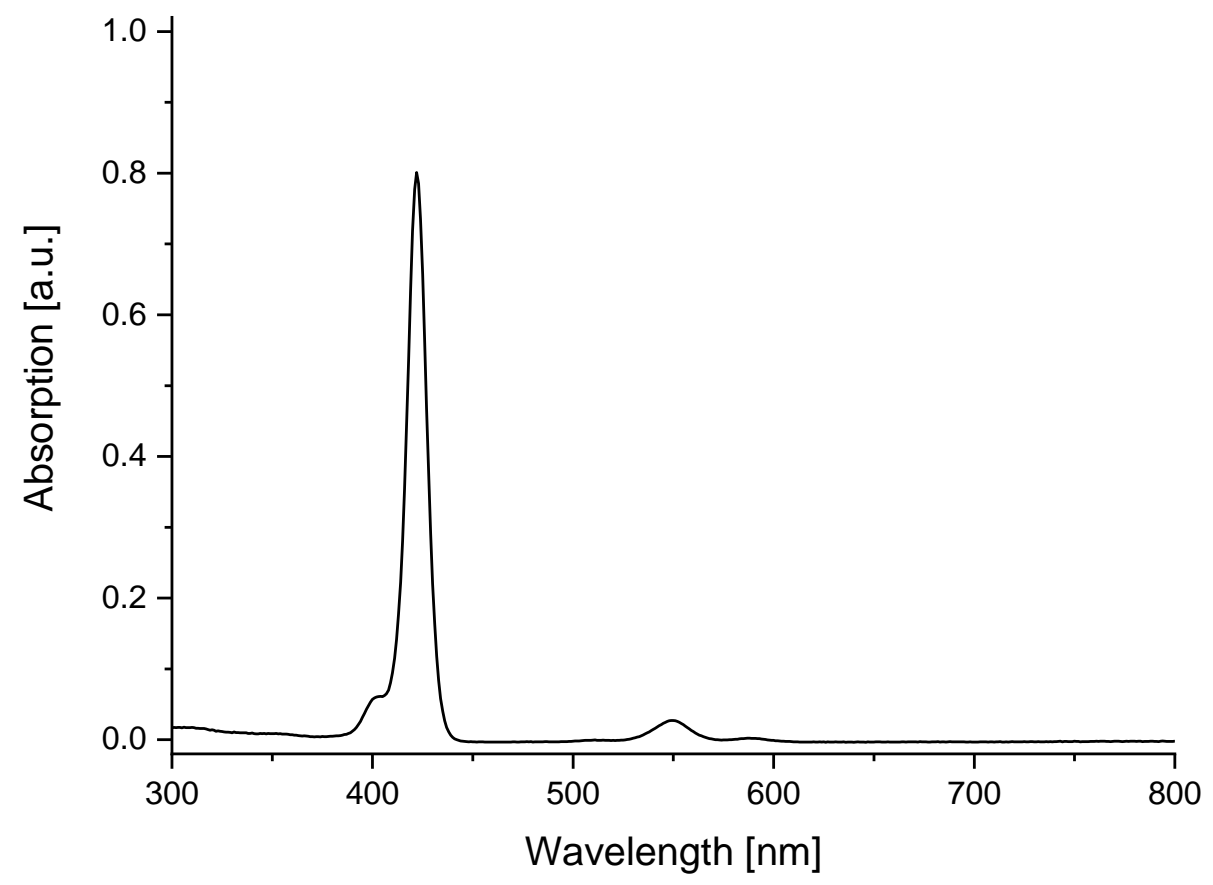

Figure S 21: Absorption spectrum of 3 measured in $\mathrm{CH}_{2} \mathrm{Cl}_{2}$. 


\section{Customer}

\begin{tabular}{|l|l|}
\hline Name: Patrick Zwick & Date:13.11.17 \\
\hline Institute: org. Chemie & Tel: 70787 \\
\hline Labor:OC8 & Email:patrick.zwick@unibas.ch \\
\hline
\end{tabular}

Email supervisor: marcel.mayor@unibas.ch

\section{Sample}

Sample name:zwp306

Aspect / texture: pinkish microcrystalline solid

\begin{tabular}{|l|l}
\hline Molecular formula: $\mathrm{C} 65 \mathrm{H} 63 \mathrm{BrN} 4 \mathrm{SiZn}$ & Molar mass: 1070.330 (exact mass)
\end{tabular}

Used solvent: DCM, $\mathrm{MeOH}$

Structure:

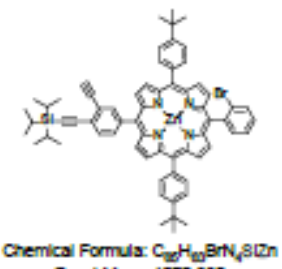

Eroct Mass: 100.330

Elemertal Nvatjala: C, 72.72; $4,5.91 ; 8 \mathrm{~B}, 7.44 ; \mathrm{N}, 5.22 ; 81,2.62 ; 2 n, 6.09$

\section{$\underline{\text { Sample details }}$}

Is the sample harmful? (skin, lungs, toxic)

$\begin{array}{ll}\text { Yes } \square & \text { No } \square \\ \text { Yes } \square & \text { No } \square \\ \text { Yes } \square & \text { No } \square \\ \text { Yes } \square & \text { No } \square \\ \text { Yes } \square & \text { No } \square\end{array}$

Is the sample light sensitive?

Is the sample electrostatic?

Is the sample hygroscopic?

Do you want your sample back?

Yes $\square$

No

Additional informations:

Figure S 22: Order of Elemental Analysis of 3. 


\section{Elemental analysis}

Sample name:zwp306

Single determination $\bigotimes$

Double determination $\square$

\begin{tabular}{|c|c|c|}
\hline 区 & $\begin{array}{ll}\mathrm{H} & \mathrm{X} \\
\end{array}$ & $\mathrm{N} \quad$ Q \\
\hline
\end{tabular}

\section{Results}

\begin{tabular}{|c|c|c|c|}
\hline \multicolumn{4}{|c|}{ Analysis number: $2017-119$} \\
\hline \multicolumn{4}{|c|}{ Sample name:zwp306 } \\
\hline Expected values: & C $72.72 \%$ & H $5.91 \%$ & $\mathrm{~N} 5.22 \%$ \\
\hline \multirow[t]{2}{*}{ Results: } & C $73.06 \% \quad-\%$ & H $6.26 \% \quad-\%$ & $\mathrm{~N} 4.97 \% \quad-\%$ \\
\hline & \multicolumn{3}{|c|}{ Other elements: $\mathrm{Br}, \mathrm{Si}, \mathrm{Zn}$} \\
\hline
\end{tabular}

Figure S 23: Results of Elemental Analysis of 3. 


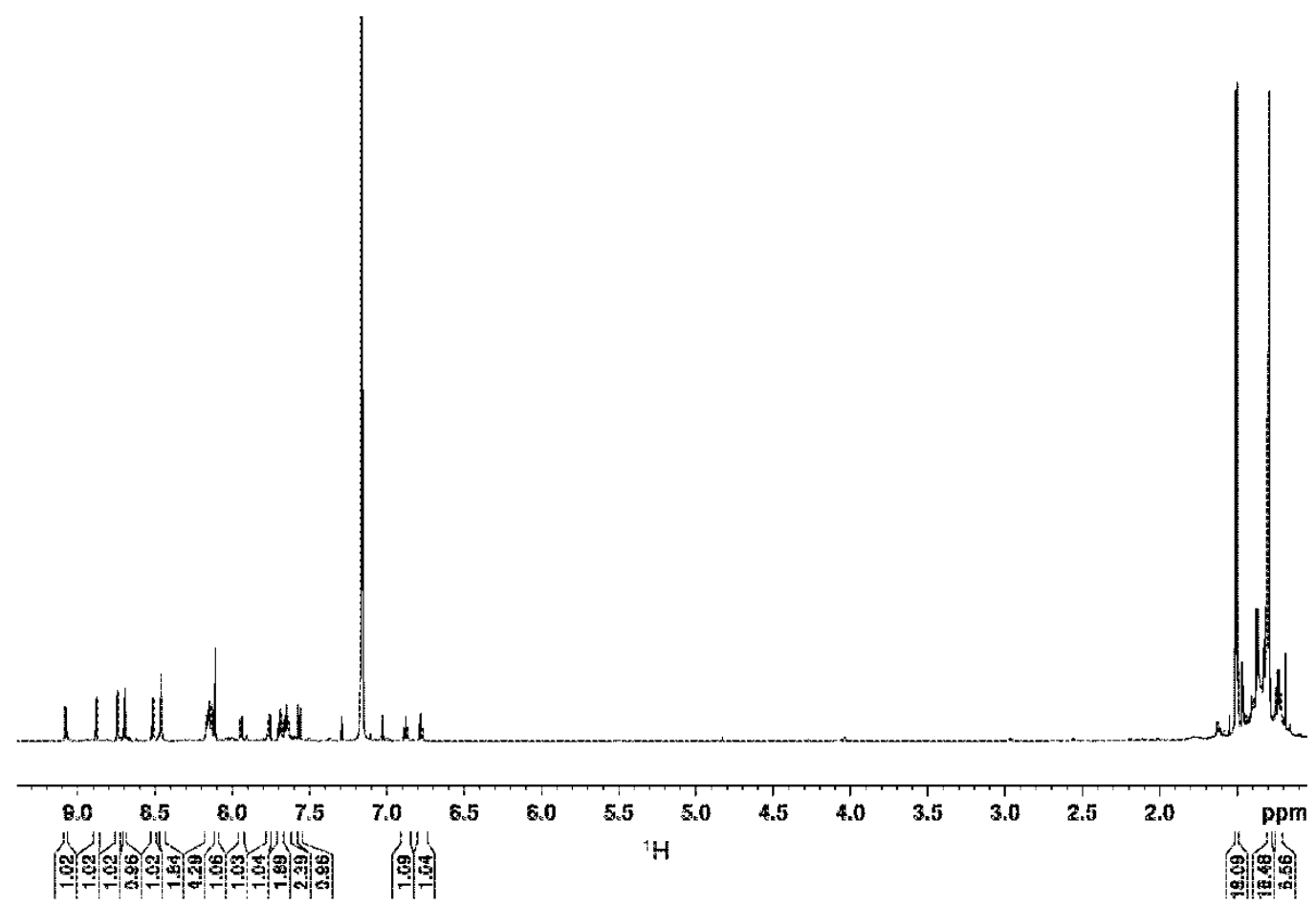

Figure S 24: ${ }^{1} \mathrm{H}-\mathrm{NMR}(600 \mathrm{MHz})$ of 2 dissolved in $\mathrm{C}_{6} \mathrm{D}_{6}$ at $298 \mathrm{~K}$. 


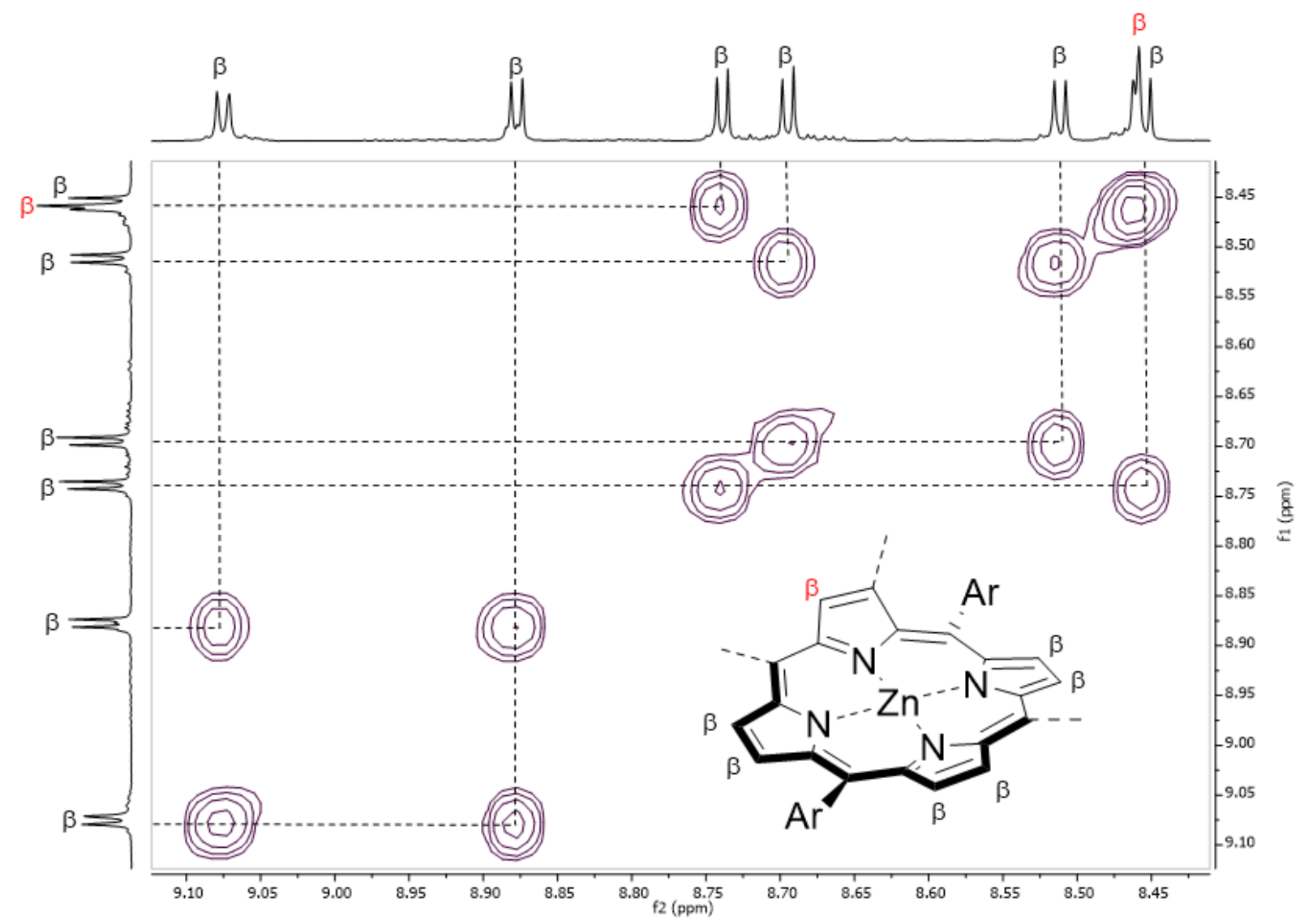

Figure S 25: Down-field aromatic region of the ${ }^{1} \mathrm{H}-{ }^{-1} \mathrm{H}$ COSY NMR spectrum of 2 in $\mathrm{C}_{6} \mathrm{D}_{6}$ highlighting the singlet $\beta$ proton next to the additional $\mathrm{C}-\mathrm{C}$ bond in red. 


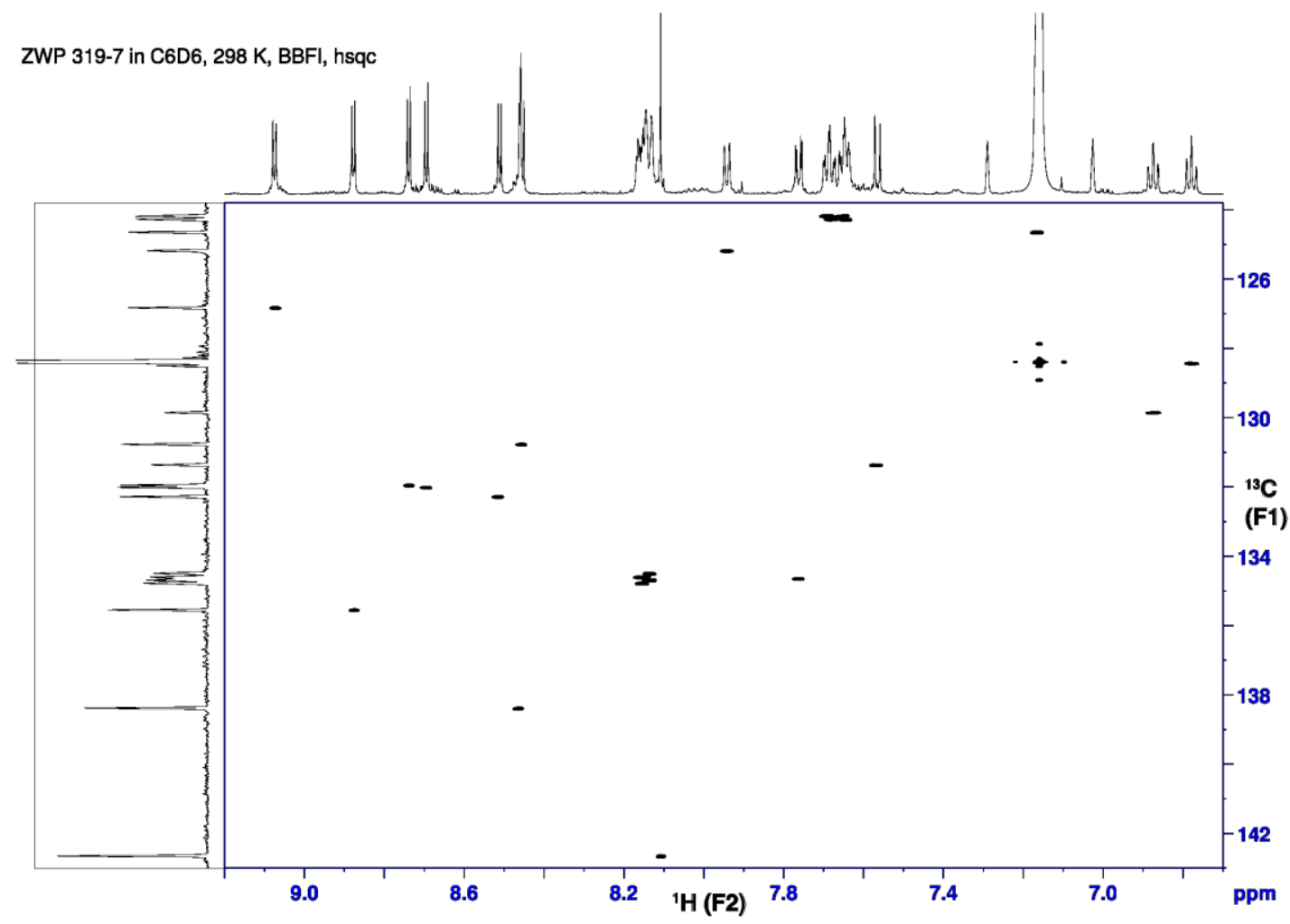

Figure S 26: Aromatic region of the ${ }^{1} \mathrm{H}^{13} \mathrm{C}-\mathrm{HSQC}$ spectrum of 2 dissolved in $\mathrm{C}_{6} \mathrm{D}_{6}$ at $298 \mathrm{~K}$. 


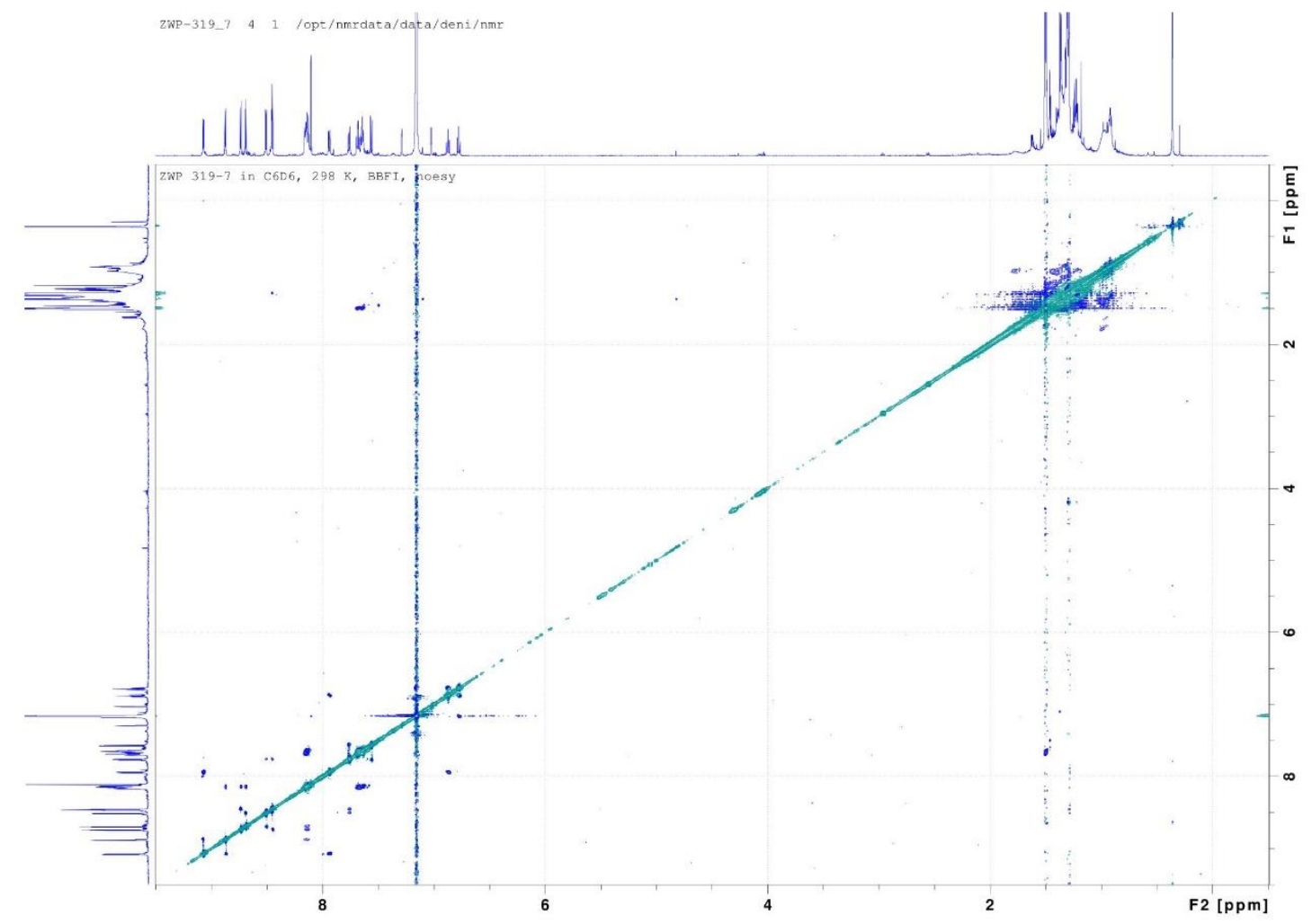

Figure S 27: ${ }^{1} \mathrm{H}-{ }^{1} \mathrm{H}-\mathrm{ROESY}$ spectrum of compound $2\left(14.1 \mathrm{~T}, \mathrm{C}_{6} \mathrm{D}_{6}, 298 \mathrm{~K}\right)$. 


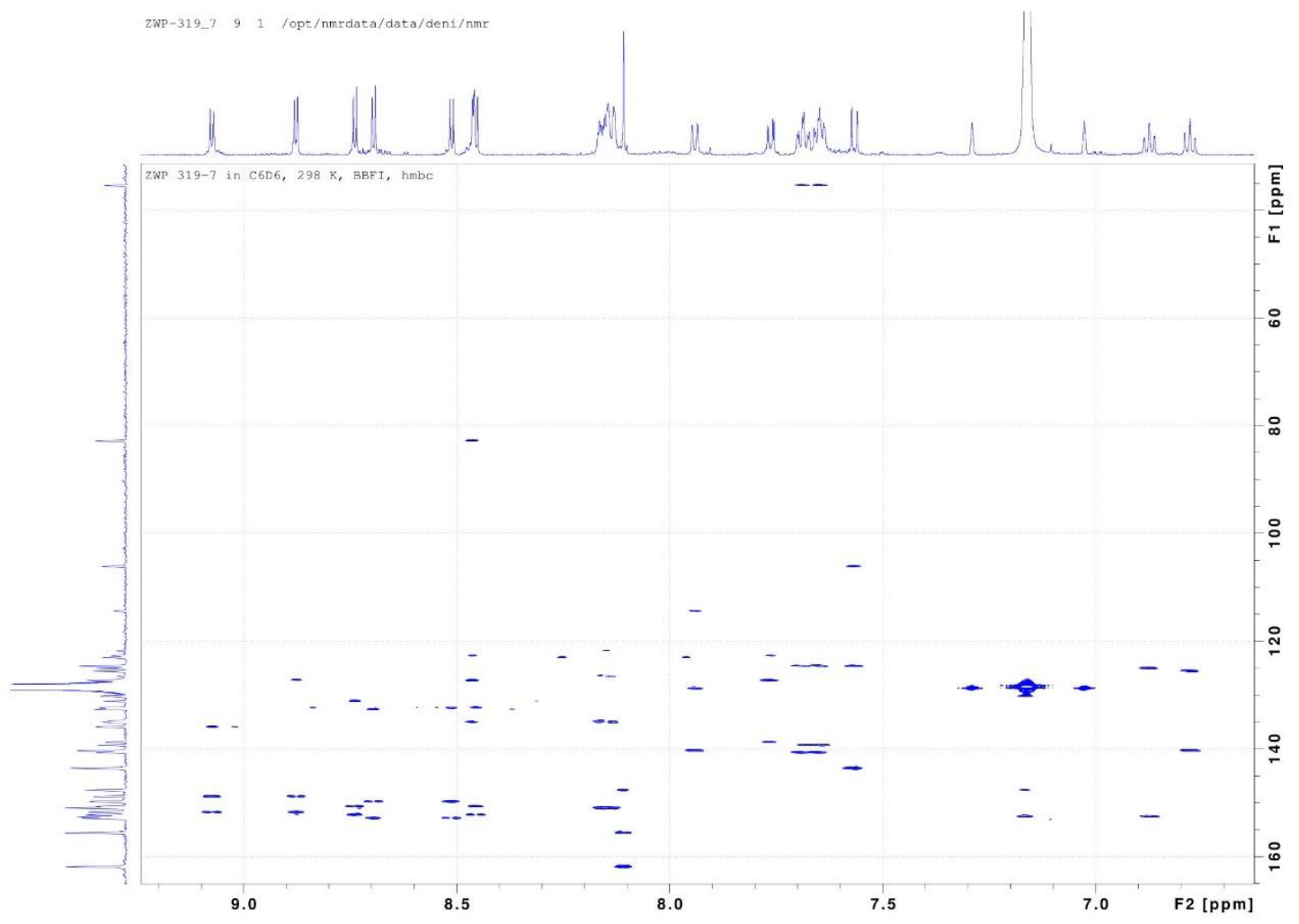

Figure $S$ 28: Aromatic region of the ${ }^{1} \mathrm{H}^{-13} \mathrm{C}-\mathrm{HMBC}$ spectrum of compound 2 (14.1 T, $\left.\mathrm{C}_{6} \mathrm{D}_{6}, 298 \mathrm{~K}\right)$. 


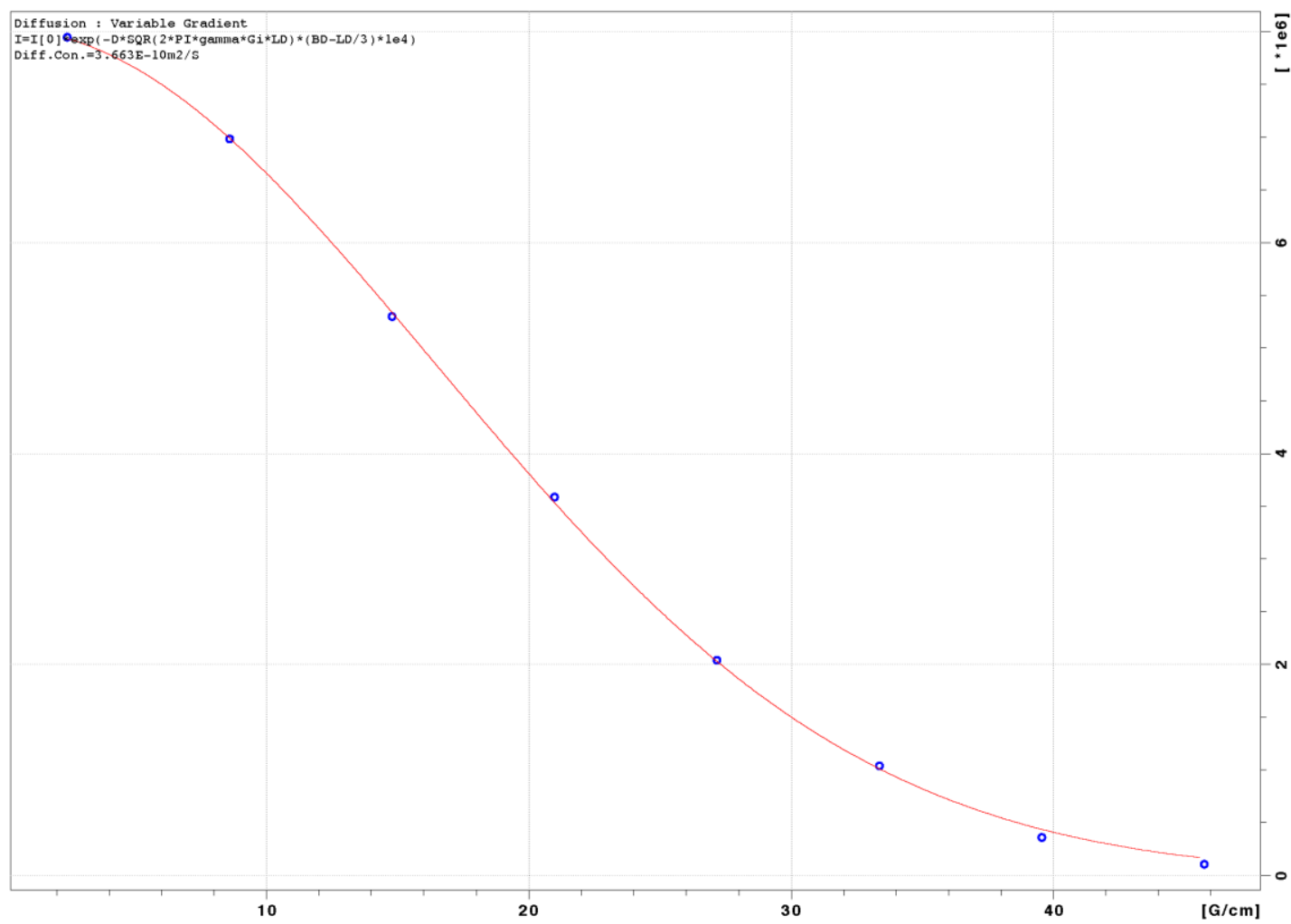

Figure S 29: Two-parameter fit for the PFGSE experiment for the determination of the diffusion coefficient of compound 2 in $\mathrm{C}_{6} \mathrm{D}_{6}$. 
Table S1: Diffusion coefficients obtained for different resonances of $\mathbf{2}$ and $\mathbf{3 .}$

\begin{tabular}{|l|l|l|l|}
\hline \multicolumn{2}{|l|}{ compound 3 } & \multicolumn{2}{l|}{ compound 2 } \\
\hline$\delta[\mathrm{ppm}]$ & $\mathrm{D}\left[10 \mathrm{e}-10 \mathrm{~m}^{2} / \mathrm{s}\right]$ & $\delta[\mathrm{ppm}]$ & $\mathrm{D}\left[10 \mathrm{e}-10 \mathrm{~m}^{2} / \mathrm{s}\right]$ \\
\hline 9.09 & 4.929 & 9.08 & 3.663 \\
\hline 8.93 & 4.965 & 8.88 & 3.582 \\
\hline 8.62 & 4.895 & 8.51 & 3.618 \\
\hline 1.51 & 4.924 & 1.51 & 3.633 \\
\hline 1.41 & 4.928 & 1.30 & 3.702 \\
\hline mean & $4.928 \pm 0.0195$ & mean & $3.640 \pm 0.0356$ \\
\hline
\end{tabular}




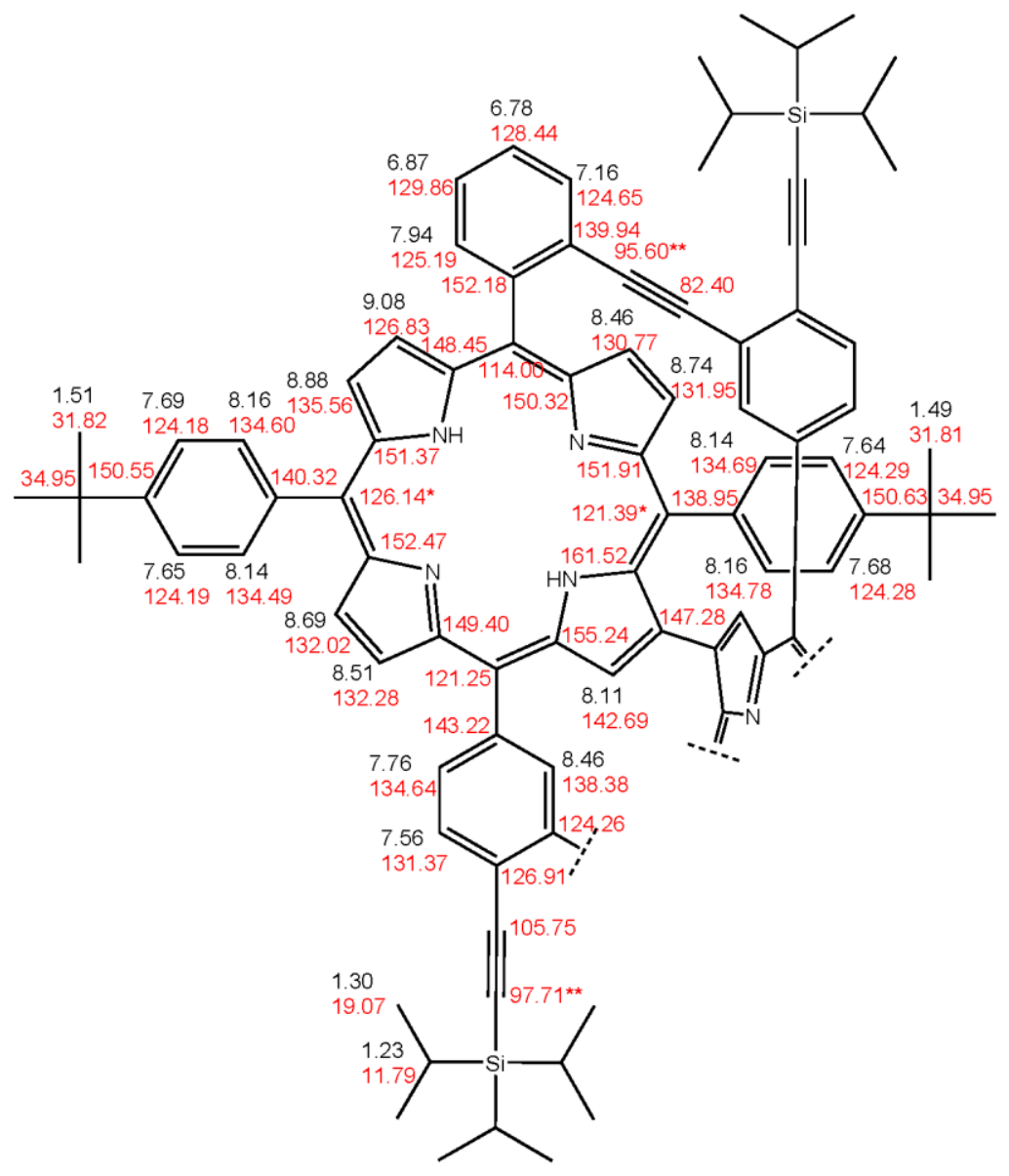

Figure S 30: Complete assignment of 2 to proton (black numbers) and carbon (red numbers) chemical shifts. The asterisks $\left(^{*}\right)$ denotes two quarternary carbon resonances that could not be assigned unambiguously due to signal overlap. The double asterisks $\left(^{* *}\right)$ indicate two alkyne resonances that displayed only very weak cross peaks in the hmbc spectra and could therefore not be assigned with certainty. 


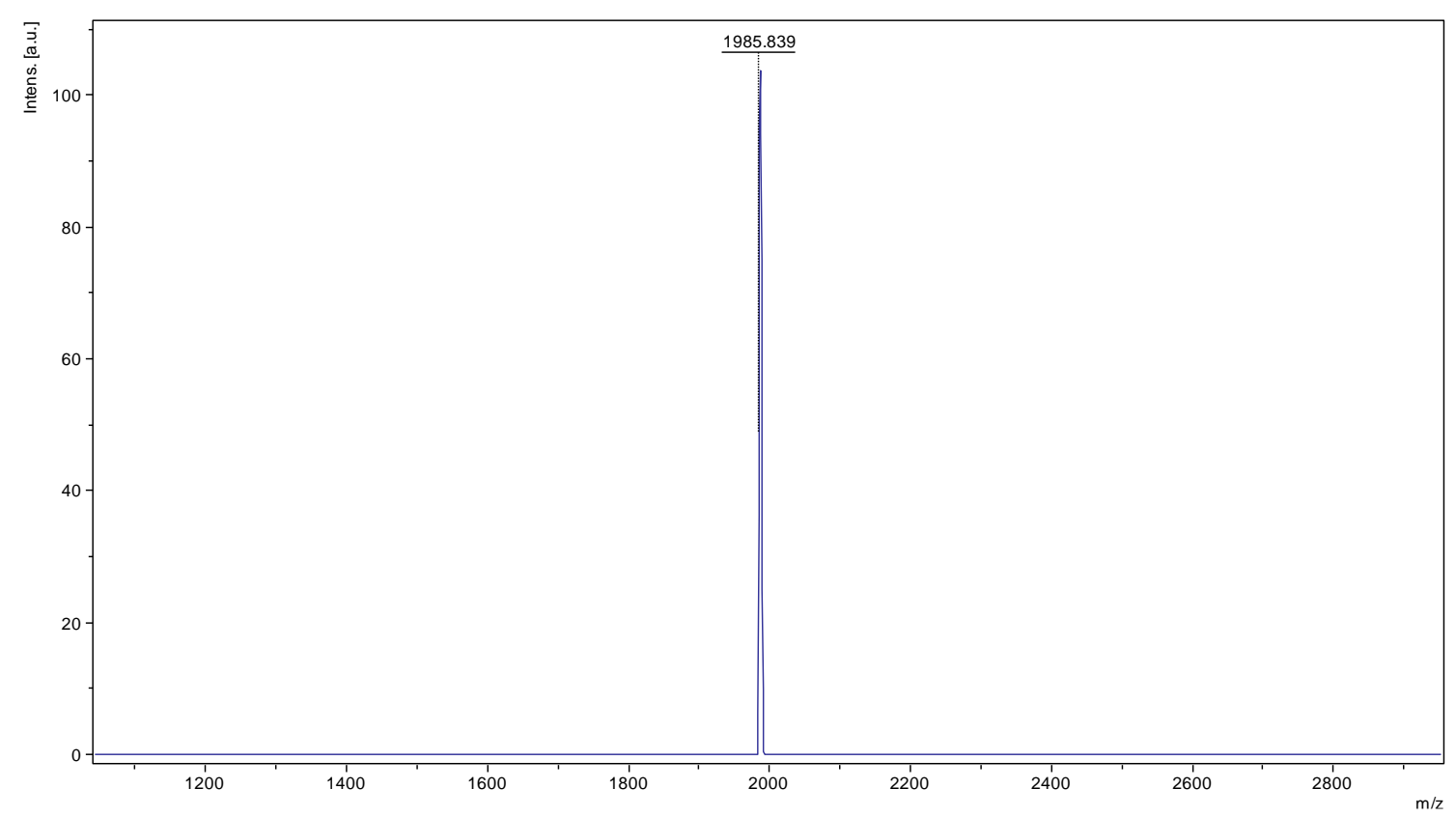

Figure S 31: MALDI-ToF mass spectrum of 2.

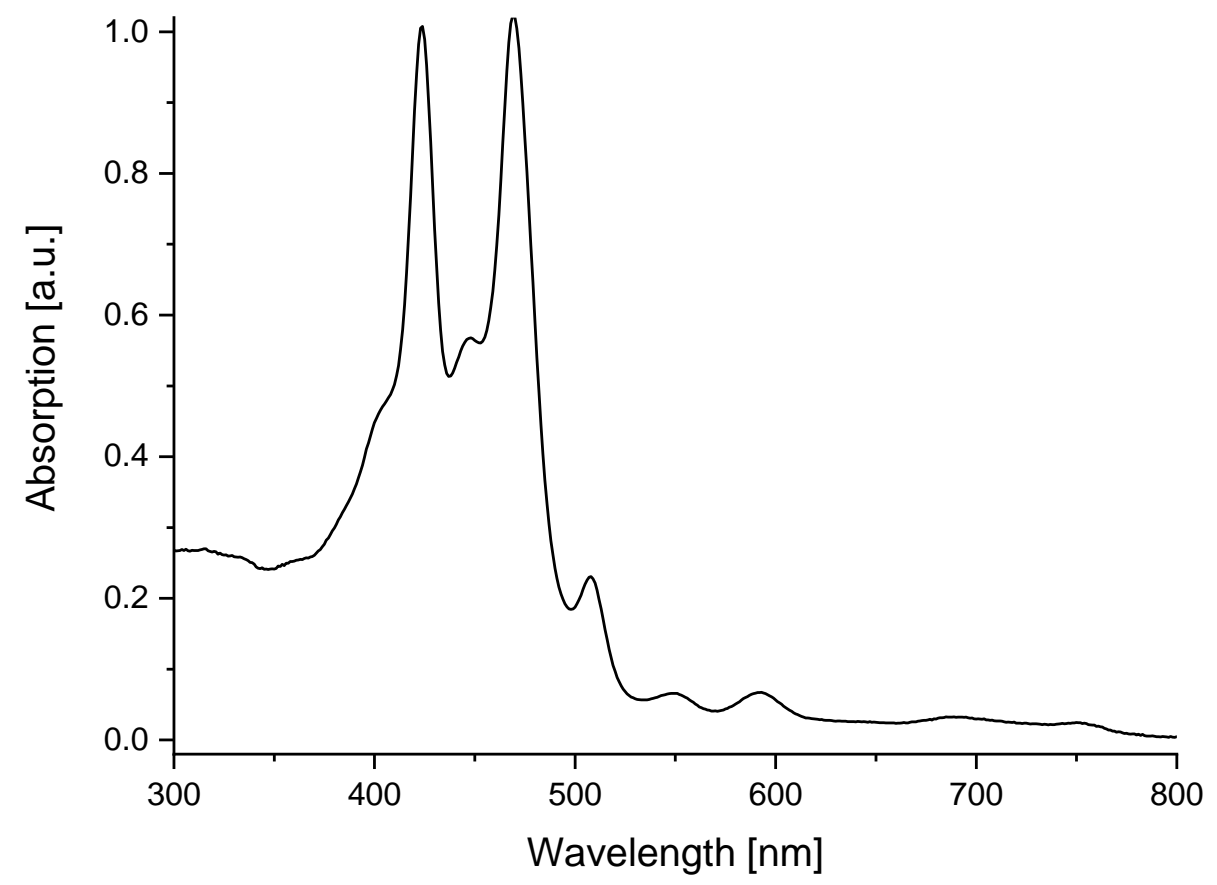

Figure $S$ 32: Absorption spectrum of 2 measured in $\mathrm{CH}_{2} \mathrm{Cl}_{2}$. 

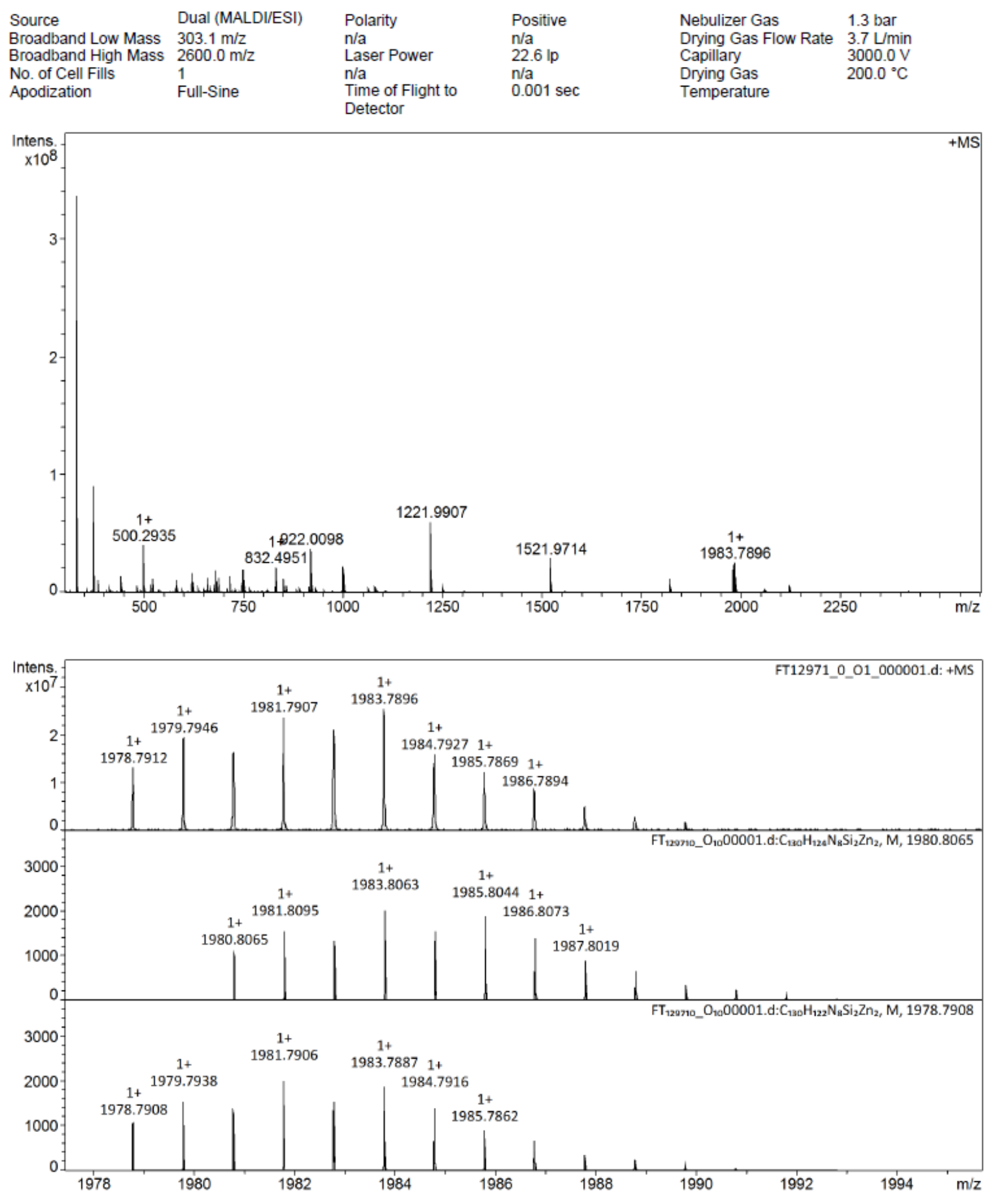

Figure S 33: HR-MS (MALDI-ToF, +) spectrum of 2. 


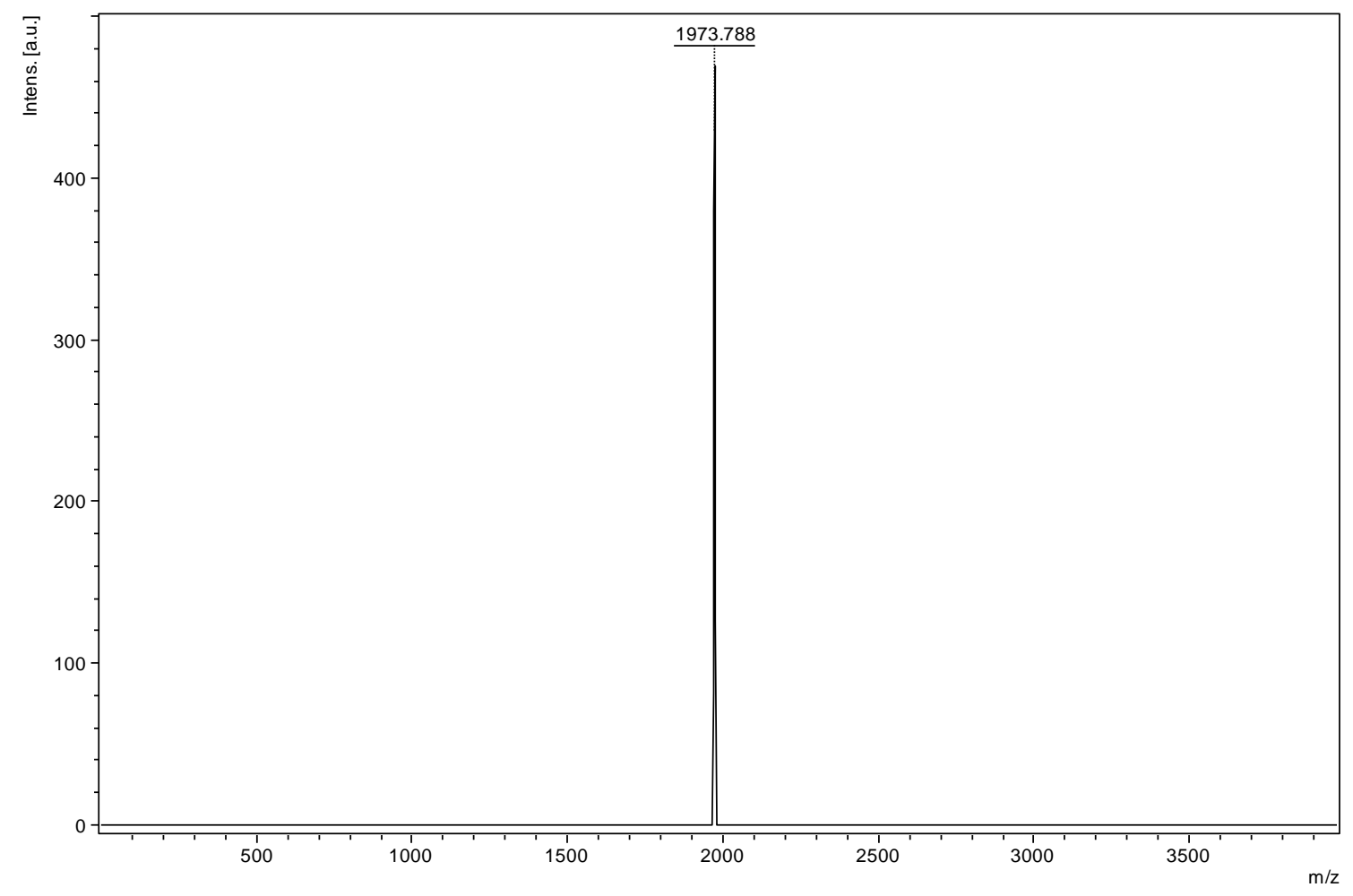

Figure S 34: MALDI-ToF mass spectrum of 1. 

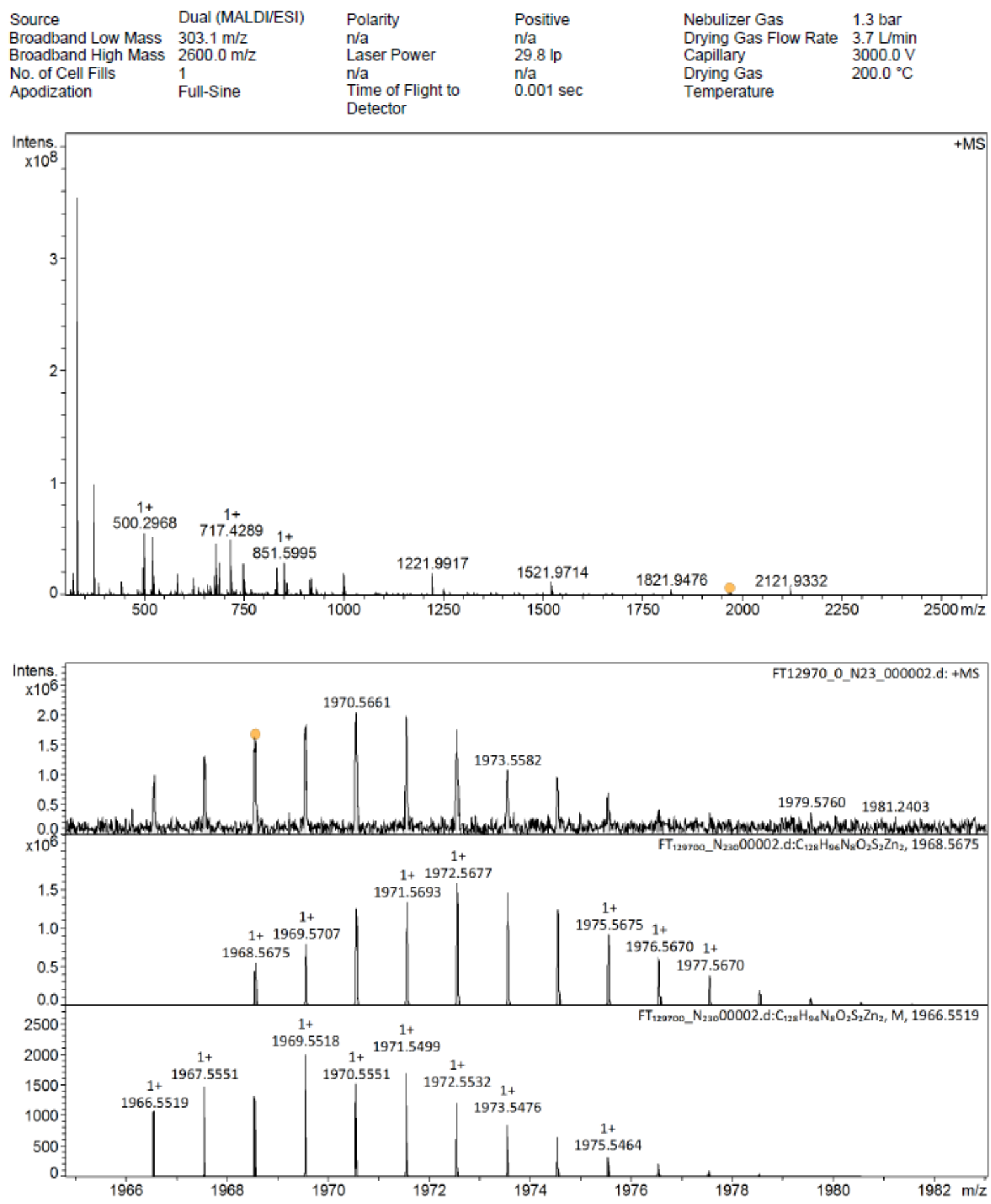

Figure S 35: HR-MS (MALDI-ToF, +) spectrum of 1. 


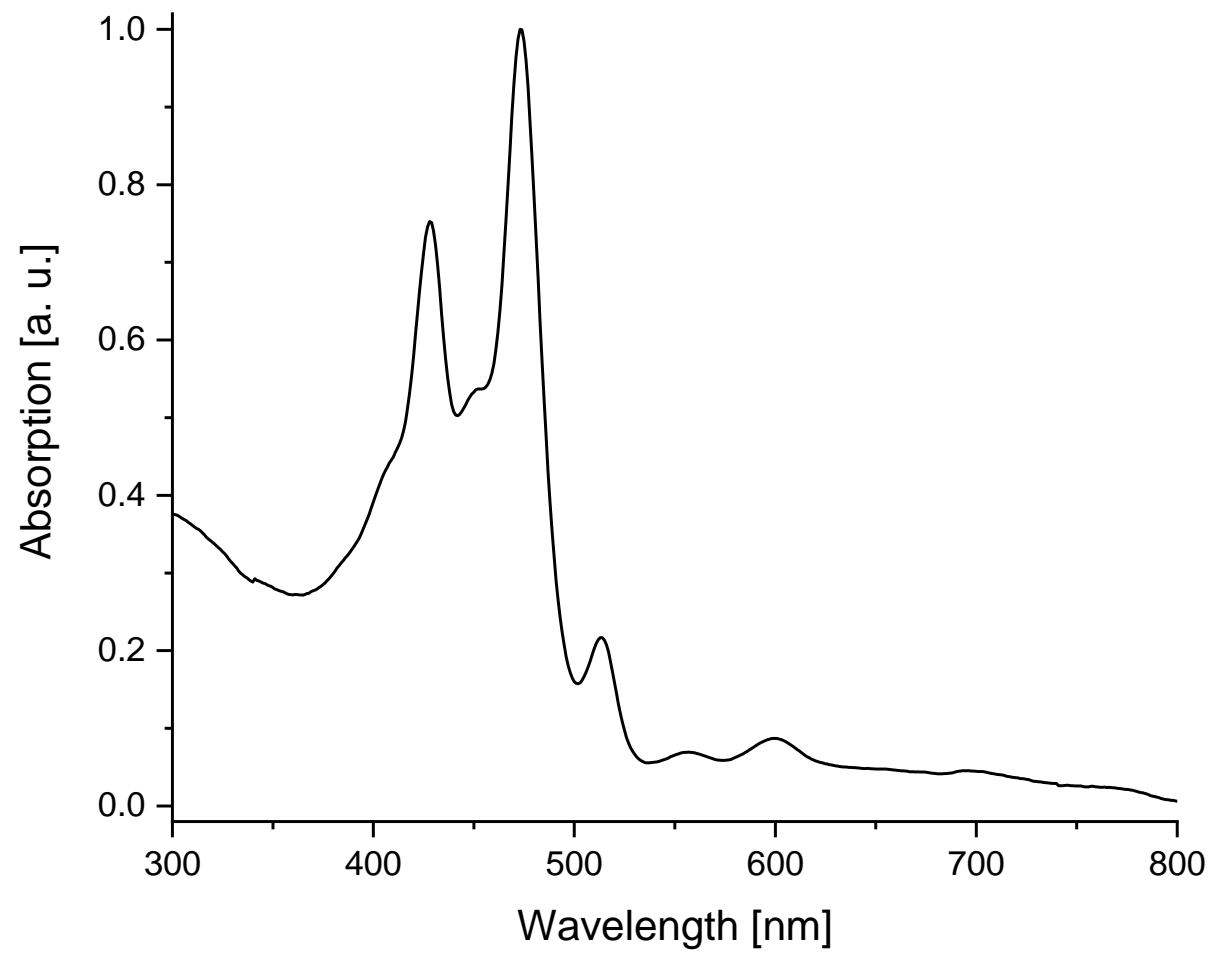

Figure $S$ 36: Absorption spectrum of 1 measured in $\mathrm{CH}_{2} \mathrm{Cl}_{2}$. 
Conductance Experiments

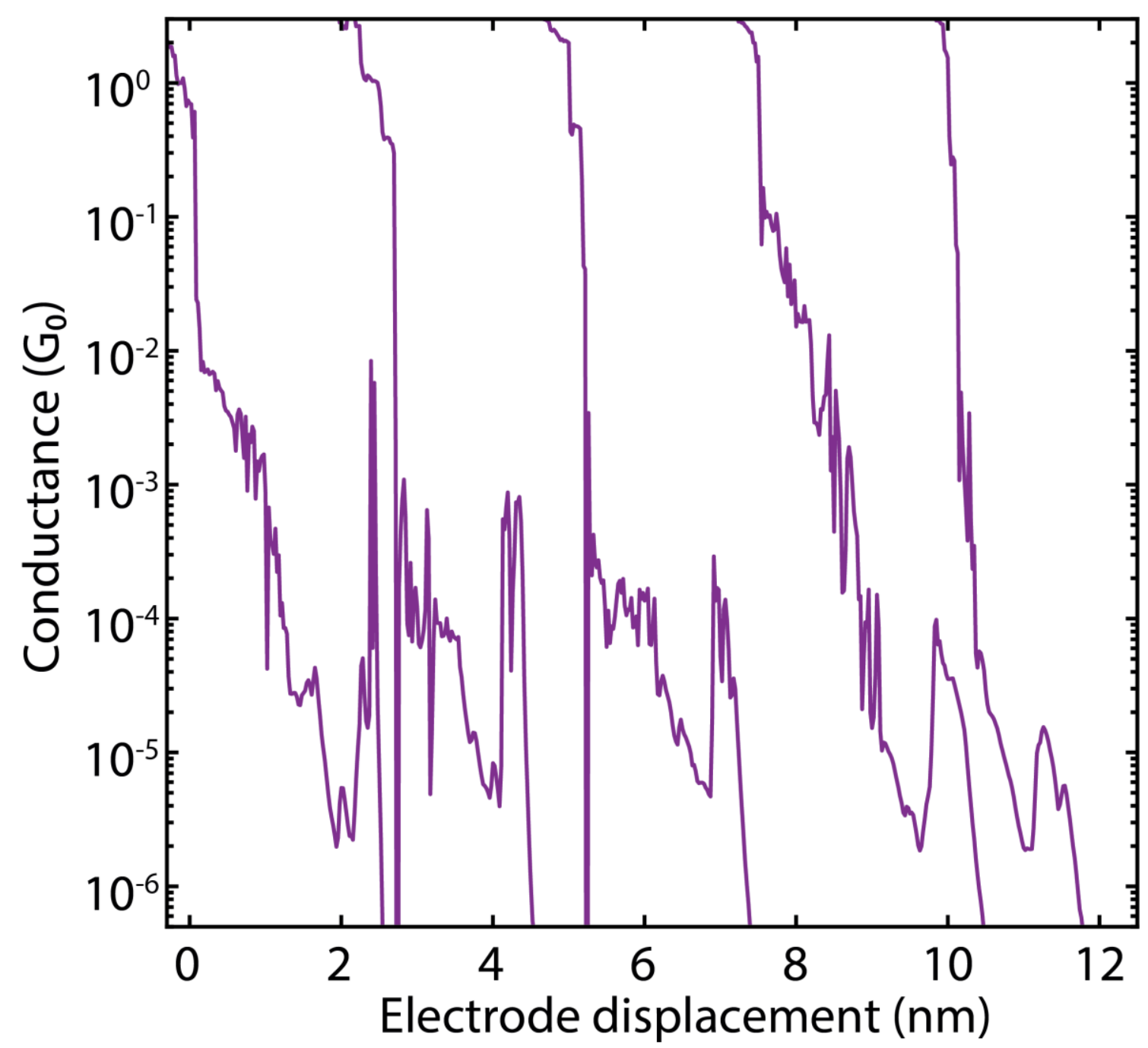

Figure S 37: Single traces that indicate conductance response to mechanical manipulation. Traces are vertically off-set for clarity. 


\section{Calculations}

Gaussian 09 (release D.01) was used for all calculations. The molecular geometry of 2 was optimized using B3LYP functional and LanL2DZ basis set. Stationary point was confirmed by subsequent frequency calculation. All vibrational frequencies were positive.

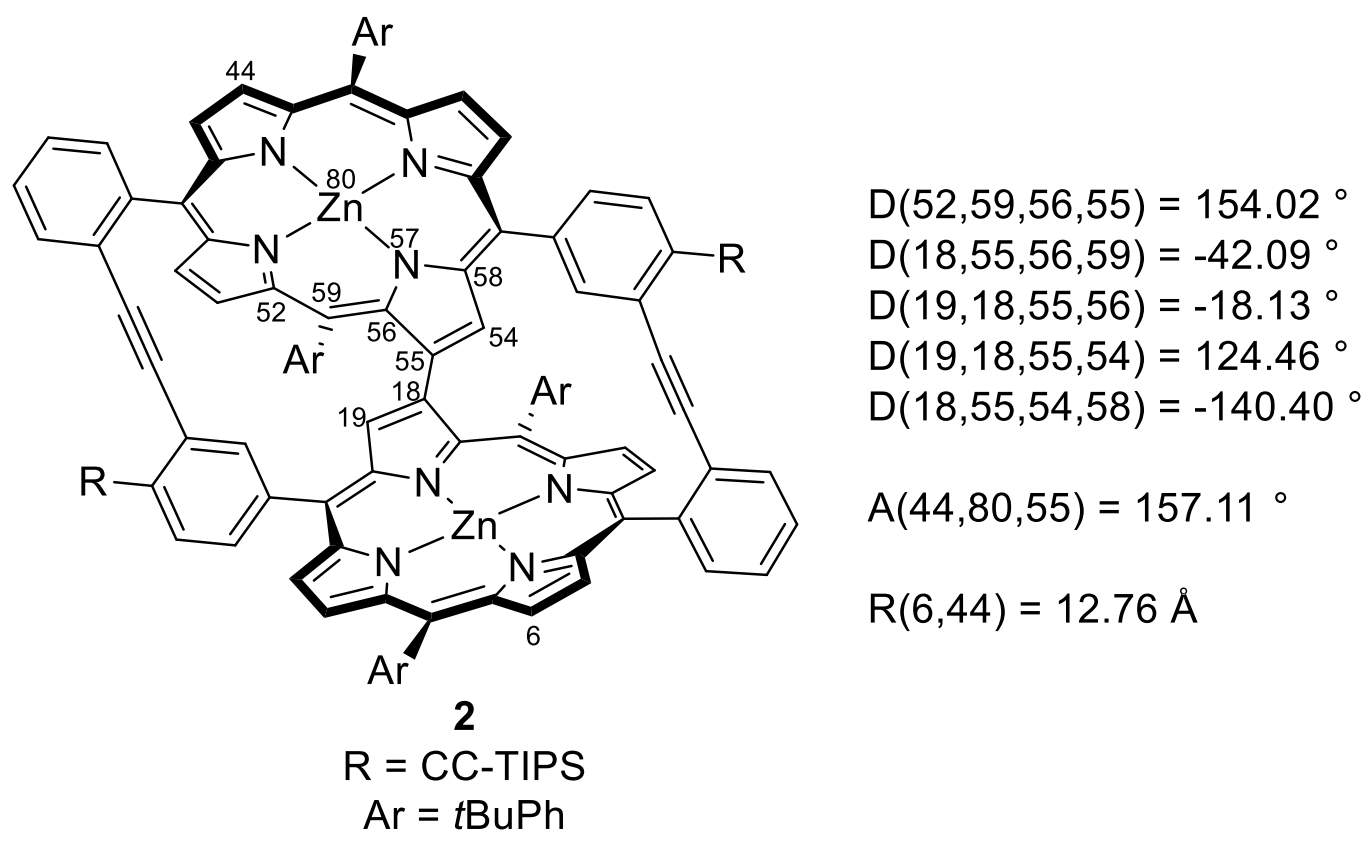

Figure S 38: Angles and distances of certain atoms in the calculated bowl-shaped structure of 2 . The curvature can be illustrated by the angle of N-Zn-N atoms of a single porphyrin $(44,80,55)$ being $157^{\circ}$ instead of usual $180^{\circ}$ for fully planar $\mathrm{sp}^{2}$-hybridized system. Moreover, the distorted porphyrin systems form a sharp angle which can be demonstrated by the dihedral angle of $\beta-\beta-\beta-\beta$ carbons $(19,18,55,54)$ being $124^{\circ}$ instead of the expected value of $180^{\circ}$ for $\mathrm{sp}^{2}$-hybridized system.

Cartesian coordinates of 2, $E_{\mathrm{el}}=-5603.45011815$ Hartree

\begin{tabular}{|c|c|c|c|}
\hline Atom & $\mathrm{X}$ & $\mathrm{Y}$ & $\mathrm{Z}$ \\
\hline $\mathrm{C}$ & 5.358779433 & 3.130553226 & 1.450176315 \\
\hline $\mathrm{C}$ & 4.621073878 & 2.612972356 & 2.489618622 \\
\hline $\mathrm{C}$ & 3.235284721 & 2.542252302 & 2.04121133 \\
\hline $\mathrm{N}$ & 3.13960369 & 3.135258272 & 0.7805549 \\
\hline $\mathrm{C}$ & 4.430956413 & 3.434024688 & 0.370644332 \\
\hline $\mathrm{C}$ & 3.084933833 & 5.648881365 & -3.635092654 \\
\hline $\mathrm{C}$ & 4.224987454 & 5.071536775 & -3.125993915 \\
\hline $\mathrm{C}$ & 3.887426923 & 4.517446684 & -1.8196629 \\
\hline
\end{tabular}




\begin{tabular}{|c|c|c|c|}
\hline $\mathrm{N}$ & 2.525503744 & 4.719444415 & -1.583251809 \\
\hline $\mathrm{C}$ & 2.014141163 & 5.424310236 & -2.671894838 \\
\hline $\mathrm{C}$ & 4.796160866 & 3.92295725 & -0.908898431 \\
\hline $\mathrm{C}$ & -2.566232577 & 4.902992156 & -1.421612246 \\
\hline $\mathrm{C}$ & -1.826236528 & 5.662529454 & -2.298784198 \\
\hline $\mathrm{C}$ & -0.420451119 & 5.357714346 & -2.061305017 \\
\hline $\mathrm{N}$ & -0.326188737 & 4.469629382 & -0.990516074 \\
\hline $\mathrm{C}$ & -1.628454346 & 4.145098417 & -0.604538122 \\
\hline $\mathrm{C}$ & 0.661651505 & 5.806604526 & -2.862184551 \\
\hline $\mathrm{C}$ & -0.12125264 & 0.711219907 & 2.325725183 \\
\hline $\mathrm{C}$ & -1.318579951 & 1.194818364 & 1.809891395 \\
\hline $\mathrm{C}$ & -1.046386862 & 2.440120922 & 1.139570388 \\
\hline $\mathrm{N}$ & 0.305667804 & 2.764656785 & 1.305117509 \\
\hline $\mathrm{C}$ & 0.862986172 & 1.788289208 & 2.126015315 \\
\hline $\mathrm{C}$ & 2.181945113 & 1.827409553 & 2.661278739 \\
\hline $\mathrm{C}$ & -1.978673524 & 3.151866198 & 0.341602333 \\
\hline $\mathrm{C}$ & 6.231455249 & 3.777613122 & -1.311836609 \\
\hline $\mathrm{C}$ & -3.433393906 & 2.768278256 & 0.439252239 \\
\hline $\mathrm{C}$ & -3.914022188 & 1.553404058 & -0.093762754 \\
\hline $\mathrm{C}$ & -5.278276744 & 1.178623578 & 0.003215053 \\
\hline $\mathrm{C}$ & -6.198777991 & 2.074085408 & 0.641462681 \\
\hline $\mathrm{C}$ & -5.710347673 & 3.296755269 & 1.161779851 \\
\hline $\mathrm{C}$ & -4.35461608 & 3.636877833 & 1.068785498 \\
\hline $\mathrm{C}$ & 6.992902358 & 4.919064374 & -1.648445207 \\
\hline $\mathrm{C}$ & 8.334762252 & 4.822778313 & -2.052502059 \\
\hline $\mathrm{C}$ & 8.947704477 & 3.557709909 & -2.133374772 \\
\hline $\mathrm{C}$ & 8.217287657 & 2.410647572 & -1.794757994 \\
\hline $\mathrm{C}$ & 6.862396658 & 2.4922575 & -1.37477799 \\
\hline $\mathrm{C}$ & 6.209725262 & 1.257788238 & -1.023565952 \\
\hline $\mathrm{C}$ & -5.743392938 & -0.08367045 & -0.497487423 \\
\hline $\mathrm{C}$ & -6.213851957 & -1.164235566 & -0.844239802 \\
\hline $\mathrm{Zn}$ & 1.411390333 & 3.83537426 & -0.090207726 \\
\hline $\mathrm{C}$ & -7.589289688 & 1.756011064 & 0.777246197 \\
\hline $\mathrm{C}$ & -8.788473854 & 1.505727924 & 0.914276928 \\
\hline $\mathrm{C}$ & -4.36546605 & -4.887093053 & -3.161892452 \\
\hline $\mathrm{C}$ & -3.245310892 & -5.426702021 & -3.750556811 \\
\hline $\mathrm{C}$ & -2.130087483 & -5.226034208 & -2.83328787 \\
\hline $\mathrm{N}$ & -2.594836353 & -4.57460572 & -1.691878752 \\
\hline $\mathrm{C}$ & -3.968906427 & -4.381065372 & -1.852869462 \\
\hline $\mathrm{C}$ & -4.514928936 & -2.660607668 & 2.560910219 \\
\hline $\mathrm{C}$ & -5.295819662 & -3.141354356 & 1.53537846 \\
\hline $\mathrm{C}$ & -4.415796192 & -3.394409188 & 0.404179639 \\
\hline $\mathrm{N}$ & -3.109137909 & -3.102190229 & 0.767432777 \\
\hline $\mathrm{C}$ & -3.151218545 & -2.56144267 & 2.054160812 \\
\hline & & & \\
\hline
\end{tabular}




\begin{tabular}{|c|c|c|c|}
\hline C & -4.836718422 & -3.832620638 & -0.876805536 \\
\hline $\mathrm{C}$ & 1.385057853 & -1.179577793 & 1.691040555 \\
\hline $\mathrm{C}$ & 0.210439109 & -0.72547557 & 2.280710001 \\
\hline $\mathrm{C}$ & -0.78133691 & -1.793548902 & 2.068148495 \\
\hline $\mathrm{N}$ & -0.258432521 & -2.725857023 & 1.175840556 \\
\hline $\mathrm{C}$ & 1.084971127 & -2.390004058 & 0.970826686 \\
\hline $\mathrm{C}$ & -2.075351058 & -1.865198565 & 2.657230205 \\
\hline $\mathrm{C}$ & 1.726385022 & -5.435717 & -2.65234195 \\
\hline $\mathrm{C}$ & 2.500555791 & -4.714680025 & -1.772307848 \\
\hline $\mathrm{C}$ & 1.59596563 & -4.003487902 & -0.879241251 \\
\hline $\mathrm{N}$ & 0.279421932 & -4.317659585 & -1.223538849 \\
\hline $\mathrm{C}$ & 0.330699113 & -5.154175187 & -2.337977382 \\
\hline $\mathrm{C}$ & -0.783931744 & -5.579620461 & -3.106436326 \\
\hline $\mathrm{C}$ & 1.983966531 & -3.058817653 & 0.100837214 \\
\hline $\mathrm{C}$ & -6.289064629 & -3.668974781 & -1.206078511 \\
\hline $\mathrm{C}$ & 3.445528342 & -2.696404922 & 0.176736672 \\
\hline $\mathrm{C}$ & 3.9392797 & -1.467492962 & -0.308497798 \\
\hline $\mathrm{C}$ & 5.314212146 & -1.12073927 & -0.225716486 \\
\hline $\mathrm{C}$ & 6.228699664 & -2.06082856 & 0.351532394 \\
\hline $\mathrm{C}$ & 5.724377097 & -3.2980798 & 0.825108989 \\
\hline $\mathrm{C}$ & 4.362623147 & -3.610271757 & 0.745319757 \\
\hline $\mathrm{C}$ & -7.080045665 & -4.790977799 & -1.538034399 \\
\hline $\mathrm{C}$ & -8.442041936 & -4.666882594 & -1.859683314 \\
\hline $\mathrm{C}$ & -9.045108486 & -3.39397881 & -1.859875833 \\
\hline $\mathrm{C}$ & -8.283227392 & -2.265038831 & -1.528371858 \\
\hline $\mathrm{C}$ & -6.906495345 & -2.37616996 & -1.193562658 \\
\hline $\mathrm{C}$ & 5.777020093 & 0.157400484 & -0.688696837 \\
\hline $\mathrm{Zn}$ & -1.418881673 & -3.742431084 & -0.21552926 \\
\hline $\mathrm{C}$ & 7.634371959 & -1.812681955 & 0.491857929 \\
\hline $\mathrm{C}$ & 8.850259344 & -1.716989862 & 0.675221525 \\
\hline $\mathrm{C}$ & 2.467270461 & 1.056734703 & 3.91064727 \\
\hline $\mathrm{C}$ & -2.310947766 & -1.152015529 & 3.950576959 \\
\hline $\mathrm{C}$ & 3.578433361 & 0.186117144 & 4.034469626 \\
\hline $\mathrm{C}$ & 3.843624441 & -0.485109674 & 5.233649756 \\
\hline $\mathrm{C}$ & 3.021181537 & -0.322165245 & 6.376351154 \\
\hline $\mathrm{C}$ & 1.907274002 & 0.534055896 & 6.244080503 \\
\hline $\mathrm{C}$ & 1.63066559 & 1.203347412 & 5.038389687 \\
\hline $\mathrm{C}$ & -1.428220897 & -1.344161494 & 5.035409044 \\
\hline $\mathrm{C}$ & -1.656789837 & -0.729591427 & 6.279707995 \\
\hline $\mathrm{C}$ & -2.76667826 & 0.114876482 & 6.495151302 \\
\hline $\mathrm{C}$ & -3.636112214 & 0.323780558 & 5.395545797 \\
\hline $\mathrm{C}$ & -3.418641074 & -0.293089015 & 4.158173865 \\
\hline $\mathrm{C}$ & -3.053621053 & 0.806163958 & 7.847216579 \\
\hline & -2.005356671 & 0.44648954 & 8.92933884 \\
\hline & & \\
\hline
\end{tabular}




\begin{tabular}{|c|c|c|c|}
\hline $\mathrm{C}$ & -4.455227343 & 0.367003858 & 8.365887625 \\
\hline $\mathrm{C}$ & -3.040393992 & 2.351443904 & 7.650981432 \\
\hline $\mathrm{C}$ & 3.360432349 & -1.072709839 & 7.683980266 \\
\hline $\mathrm{C}$ & 3.332292113 & -2.607887769 & 7.42129386 \\
\hline $\mathrm{C}$ & 4.784126203 & -0.660311861 & 8.162955356 \\
\hline $\mathrm{C}$ & 2.359240878 & -0.757952694 & 8.823188131 \\
\hline $\mathrm{C}$ & -0.526372721 & -6.409725032 & -4.330571612 \\
\hline $\mathrm{C}$ & -1.043488487 & -7.722187157 & -4.43464216 \\
\hline $\mathrm{C}$ & -0.811061821 & -8.503037941 & -5.576662401 \\
\hline $\mathrm{C}$ & -0.06234447 & -8.011625214 & -6.674360301 \\
\hline $\mathrm{C}$ & 0.44372634 & -6.697471972 & -6.567622313 \\
\hline $\mathrm{C}$ & 0.219796275 & -5.91368579 & -5.42009279 \\
\hline $\mathrm{C}$ & 0.166195668 & -8.905146908 & -7.915083713 \\
\hline $\mathrm{C}$ & -1.209440725 & -9.29432218 & -8.533322999 \\
\hline $\mathrm{C}$ & 0.921467011 & -10.19881289 & -7.488269171 \\
\hline $\mathrm{C}$ & 1.00343645 & -8.194626057 & -9.007724826 \\
\hline $\mathrm{C}$ & 0.356548128 & 6.693575153 & -4.034382888 \\
\hline $\mathrm{C}$ & 0.883520166 & 8.000043386 & -4.107123864 \\
\hline $\mathrm{C}$ & 0.607288839 & 8.838715708 & -5.203404969 \\
\hline $\mathrm{C}$ & -0.198154524 & 8.404860604 & -6.279078626 \\
\hline $\mathrm{C}$ & -0.715845705 & 7.088100394 & -6.204023813 \\
\hline $\mathrm{C}$ & -0.449911227 & 6.252148838 & -5.10939111 \\
\hline $\mathrm{C}$ & -0.522320044 & 9.293331777 & -7.501969698 \\
\hline $\mathrm{C}$ & -2.064606116 & 9.482912088 & -7.609009531 \\
\hline $\mathrm{C}$ & 0.131167332 & 10.69435596 & -7.40300576 \\
\hline $\mathrm{C}$ & 0.001007107 & 8.603146192 & -8.79654056 \\
\hline $\mathrm{Si}$ & -10.58531359 & 1.110132864 & 1.13096386 \\
\hline $\mathrm{C}$ & -10.71657275 & -0.463386283 & 2.245265497 \\
\hline $\mathrm{C}$ & -11.43206905 & 2.645987463 & 1.923933255 \\
\hline $\mathrm{C}$ & -11.3155222 & 0.688723479 & -0.609265942 \\
\hline $\mathrm{C}$ & -9.629382787 & -0.555036306 & 3.348484852 \\
\hline $\mathrm{C}$ & -12.12934245 & -0.648093093 & 2.864055366 \\
\hline $\mathrm{C}$ & -11.34338587 & 3.90488914 & 1.020151049 \\
\hline $\mathrm{C}$ & -10.86919647 & 2.972028499 & 3.333261336 \\
\hline $\mathrm{C}$ & -10.69447658 & 1.501655093 & -1.775992176 \\
\hline $\mathrm{C}$ & -12.86533699 & 0.78409845 & -0.652766626 \\
\hline $\mathrm{Si}$ & 10.66914165 & -1.717784807 & 1.038114659 \\
\hline $\mathrm{C}$ & 11.48359829 & -0.202988278 & 0.179491986 \\
\hline $\mathrm{C}$ & 10.87562781 & -1.66669075 & 2.960199017 \\
\hline $\mathrm{C}$ & 11.38050783 & -3.395526161 & 0.391444589 \\
\hline $\mathrm{C}$ & 11.34817054 & -0.254546674 & -1.36577013 \\
\hline $\mathrm{C}$ & 10.93941438 & 1.146259297 & 0.719940589 \\
\hline $\mathrm{C}$ & 12.30323086 & -1.245481243 & 3.404902543 \\
\hline & 9.810583418 & -0.80896392 & 3.693524468 \\
\hline & & \\
\hline
\end{tabular}




\begin{tabular}{|c|c|c|c|}
\hline C & 10.73077439 & -3.900462173 & -0.924086264 \\
\hline C & 12.92791127 & -3.383591234 & 0.255194679 \\
\hline$H$ & 6.424091337 & 3.308440915 & 1.422870825 \\
\hline$H$ & 4.981214926 & 2.307464847 & 3.459720716 \\
\hline$H$ & 2.968244386 & 6.145133818 & -4.587130661 \\
\hline$H$ & 5.194743035 & 5.015981526 & -3.596853071 \\
\hline$H$ & -3.642342848 & 4.855656618 & -1.34227595 \\
\hline$H$ & -2.196291653 & 6.349056888 & -3.045265279 \\
\hline$H$ & -2.240191265 & 0.64630675 & 1.725527594 \\
\hline$H$ & -3.227762557 & 0.87766885 & -0.59620481 \\
\hline$H$ & -6.408545614 & 3.971995336 & 1.648483245 \\
\hline$H$ & -3.998487644 & 4.572246757 & 1.492257496 \\
\hline$H$ & 6.523545205 & 5.895834159 & -1.571670294 \\
\hline$H$ & 8.894468902 & 5.722268559 & -2.29631116 \\
\hline$H$ & 9.983421279 & 3.467424864 & -2.450517857 \\
\hline$H$ & 8.682050963 & 1.431269582 & -1.849770173 \\
\hline$H$ & -5.358902756 & -4.827289859 & -3.580188239 \\
\hline$H$ & -3.170680682 & -5.882556126 & -4.726746236 \\
\hline$H$ & -4.832564074 & -2.397477313 & 3.557901447 \\
\hline$H$ & -6.36034166 & -3.325789092 & 1.548582887 \\
\hline$H$ & 2.302234979 & -0.625422616 & 1.5944891 \\
\hline$H$ & 2.066369548 & -6.082627922 & -3.446923754 \\
\hline$H$ & 3.578903954 & -4.663903818 & -1.73732823 \\
\hline$H$ & 3.256435826 & -0.755927931 & -0.764086317 \\
\hline$H$ & 6.419814327 & -4.006997642 & 1.265541687 \\
\hline$H$ & 3.998254273 & -4.558432512 & 1.131627775 \\
\hline$H$ & -6.618383377 & -5.774373469 & -1.52174581 \\
\hline$H$ & -9.025489002 & -5.551534242 & -2.102148379 \\
\hline$H$ & -10.09675665 & -3.284998483 & -2.112958308 \\
\hline$H$ & -8.737163265 & -1.278079208 & -1.524616 \\
\hline$H$ & 4.222404647 & 0.012714987 & 3.176454559 \\
\hline$H$ & 4.701663369 & -1.151867202 & 5.272865595 \\
\hline$H$ & 1.240559202 & 0.696487016 & 7.08421761 \\
\hline$H$ & 0.766694342 & 1.859273065 & 4.976525462 \\
\hline$H$ & -0.565548455 & -1.992549486 & 4.909291283 \\
\hline$H$ & -0.9553388 & -0.92535119 & 7.083666542 \\
\hline$H$ & -4.493797655 & 0.984056717 & 5.499195515 \\
\hline$H$ & -4.098098053 & -0.085502928 & 3.335834402 \\
\hline$H$ & -1.982430463 & -0.632289853 & 9.131425137 \\
\hline$H$ & -0.995264455 & 0.765232781 & 8.639897372 \\
\hline$H$ & -2.256059889 & 0.955886074 & 9.868650734 \\
\hline$H$ & -5.250180373 & 0.627495066 & 7.65699198 \\
\hline$H$ & -4.489847275 & -0.718481025 & 8.525564773 \\
\hline & -4.679719025 & 0.86254688 & 9.320344172 \\
\hline & & & \\
\hline$H$
\end{tabular}




\begin{tabular}{|c|c|c|c|}
\hline$H$ & -3.791641536 & 2.674020501 & 6.920321163 \\
\hline$H$ & -3.255902519 & 2.857410087 & 8.602038752 \\
\hline$H$ & -2.059462648 & 2.691955521 & 7.29489573 \\
\hline$H$ & 4.051458373 & -2.900181751 & 6.646906864 \\
\hline$H$ & 3.584387176 & -3.155545762 & 8.339708684 \\
\hline$H$ & 2.336055792 & -2.929877398 & 7.091431256 \\
\hline$H$ & 5.548071878 & -0.891600674 & 7.411131416 \\
\hline$H$ & 4.830054096 & 0.417061479 & 8.36817429 \\
\hline$H$ & 5.045498813 & -1.197661885 & 9.084857343 \\
\hline$H$ & 1.336656899 & -1.061081336 & 8.562252734 \\
\hline$H$ & 2.646224271 & -1.308547208 & 9.728251232 \\
\hline$H$ & 2.349431441 & 0.311013648 & 9.072838471 \\
\hline$H$ & -1.61821244 & -8.13287838 & -3.607912634 \\
\hline$H$ & -1.220283216 & -9.510212876 & -5.607134598 \\
\hline$H$ & 1.016719715 & -6.265518669 & -7.38156892 \\
\hline$H$ & 0.613337133 & -4.900941502 & -5.378368962 \\
\hline$H$ & -1.837957545 & -9.836632029 & -7.816710102 \\
\hline$H$ & -1.760022069 & -8.400597105 & -8.854369735 \\
\hline$H$ & -1.063141687 & -9.940999051 & -9.409227387 \\
\hline$H$ & 1.901172893 & -9.954805546 & -7.057554334 \\
\hline$H$ & 0.358761359 & -10.76956828 & -6.739765492 \\
\hline$H$ & 1.080923197 & -10.85032401 & -8.358269602 \\
\hline$H$ & 2.000959919 & -7.919037917 & -8.641511097 \\
\hline$H$ & 1.138613155 & -8.868141081 & -9.863731806 \\
\hline$H$ & 0.507442643 & -7.286717006 & -9.37492053 \\
\hline$H$ & 1.503266385 & 8.367914771 & -3.292726107 \\
\hline$H$ & 1.028909949 & 9.838539163 & -5.20568713 \\
\hline$H$ & -1.333642193 & 6.701841472 & -7.011426471 \\
\hline$H$ & -0.853670314 & 5.242827377 & -5.092332878 \\
\hline$H$ & -2.460129038 & 9.978754236 & -6.71311095 \\
\hline$H$ & -2.311765678 & 10.10212534 & -8.48211053 \\
\hline$H$ & -2.58488919 & 8.523878976 & -7.719297484 \\
\hline$H$ & 1.22605847 & 10.63058302 & -7.354658121 \\
\hline$H$ & -0.127892149 & 11.28467615 & -8.291286628 \\
\hline$H$ & -0.221824406 & 11.24706045 & -6.522626671 \\
\hline$H$ & 1.089196537 & 8.465652446 & -8.753209379 \\
\hline$H$ & -0.456027612 & 7.617381938 & -8.944772595 \\
\hline$H$ & -0.233111827 & 9.218097443 & -9.676200741 \\
\hline$H$ & -10.54428046 & -1.308389202 & 1.556298575 \\
\hline$H$ & -12.49866595 & 2.389698459 & 2.039635916 \\
\hline$H$ & -11.04740171 & -0.370071073 & -0.773575608 \\
\hline$H$ & -8.619849672 & -0.4819348 & 2.927279714 \\
\hline$H$ & -9.737675925 & 0.24432032 & 4.093285905 \\
\hline & -9.708205562 & -1.514800726 & 3.880575173 \\
\hline & & \\
\hline$H$ & & \\
\hline$H$
\end{tabular}




\begin{tabular}{|c|c|c|c|}
\hline$H$ & -12.17731837 & -1.590814788 & 3.428201057 \\
\hline$H$ & -12.37025363 & 0.163931373 & 3.563666467 \\
\hline$H$ & -12.92088423 & -0.679642419 & 2.104178441 \\
\hline$H$ & -11.83673684 & 3.758086105 & 0.051292669 \\
\hline$H$ & -10.29752331 & 4.178874561 & 0.825852225 \\
\hline$H$ & -11.82628833 & 4.762035226 & 1.512159766 \\
\hline$H$ & -11.36811534 & 3.861018219 & 3.746488147 \\
\hline$H$ & -9.792546894 & 3.185673337 & 3.288839094 \\
\hline$H$ & -11.01866613 & 2.148332464 & 4.04228982 \\
\hline$H$ & -11.08324342 & 1.143167359 & -2.740857315 \\
\hline$H$ & -10.93829996 & 2.569223981 & -1.699632402 \\
\hline$H$ & -9.601465132 & 1.415732383 & -1.796002872 \\
\hline$H$ & -13.34703982 & 0.150320192 & 0.102861942 \\
\hline$H$ & -13.20746557 & 1.815263632 & -0.490919319 \\
\hline$H$ & -13.23971798 & 0.466366116 & -1.636816869 \\
\hline$H$ & 12.55867443 & -0.251333369 & 0.421573174 \\
\hline$H$ & 10.72461217 & -2.711401042 & 3.283388822 \\
\hline$H$ & 11.1244597 & -4.127256908 & 1.177371988 \\
\hline$H$ & 11.78934413 & 0.646190995 & -1.818214818 \\
\hline$H$ & 10.29415284 & -0.302252972 & -1.671382498 \\
\hline$H$ & 11.85771292 & -1.124753461 & -1.797668698 \\
\hline$H$ & 11.41528644 & 1.988178032 & 0.195632373 \\
\hline$H$ & 9.856109404 & 1.230663759 & 0.564873125 \\
\hline$H$ & 11.13546703 & 1.271577331 & 1.792017734 \\
\hline$H$ & 12.39294528 & -1.301947147 & 4.4995006 \\
\hline$H$ & 12.52523514 & -0.21028469 & 3.112152912 \\
\hline$H$ & 13.08395674 & -1.887895992 & 2.977885433 \\
\hline$H$ & 8.791676431 & -1.118704547 & 3.432947661 \\
\hline$H$ & 9.905566967 & 0.255963375 & 3.444788328 \\
\hline$H$ & 9.92918873 & -0.905305045 & 4.783150069 \\
\hline$H$ & 10.96116836 & -3.240062404 & -1.770183812 \\
\hline$H$ & 9.639282961 & -3.960980739 & -0.840526631 \\
\hline$H$ & 11.10961981 & -4.902403505 & -1.175278106 \\
\hline$H$ & 13.29117135 & -4.376636603 & -0.046869663 \\
\hline$H$ & 13.2574091 & -2.668540341 & -0.511000187 \\
\hline$H$ & 13.43110825 & -3.121712499 & 1.194749481 \\
\hline & & & \\
\hline$H$ & & \\
\hline$H$ & & \\
\hline$H$ &
\end{tabular}

